\title{
Correlation signal subset based stochastic subspace identification for an online identification of railway vehicle suspension systems
}

\author{
Fulong Liu ${ }^{\mathrm{b}}$, Hao Zhang ${ }^{\mathrm{a}}$, Xiaocong He ${ }^{\mathrm{c}}$, Yunshi Zhao ${ }^{\mathrm{d}}$, Fengshou Gu ${ }^{\mathrm{b}}$, Andrew D. Ball ${ }^{\mathrm{b}}$ \\ ${ }^{a}$ School of Mechanical Engineering, Hebei University of Technology, Tianjin, China \\ ${ }^{b}$ Centre for Efficiency and Performance Engineering (CEPE), School of Computing and \\ Engineering, University of Huddersfield, Huddersfield, UK \\ ${ }^{c}$ Innovative Manufacturing Research Centre, Kunming University of Science and Technology, \\ Kunming, People's Republic of China \\ ${ }^{d}$ Traction Power State Key Laboratory, Southwest Jiaotong University, ChengDu, People's \\ Republic of China
}

\begin{abstract}
Monitoring the condition of suspension systems is significant to ensure the safe operation of modern railway vehicles. For this purpose, an online modal identification scheme, denoted as Correlation Subset based Stochastic Subspace Identification (CoS-SSI) is proposed in this paper to monitor the suspension conditions. Because the widespread of the dynamic contact status between wheel and track, especially under faulty suspension cases, the vibration responses measured online exhibit high nonstationarity and nonlinearity. To take into account these characteristics of signals, the input correlation signals for SSI are clustered into several successive subsets according to their magnitudes, on which SSI is implemented one by one. In this way it yields a magnitude adaptive SSI for more reliable and accurate identification. Experimental studies were conducted on a $1 / 5$ th scaled roller rig system to verify the effectiveness of the proposed method for suspension monitoring. The experimental results show that the CoS-SSI outperform the conventional SSI in that it produces more reliable and realistic identification for the nonlinear system. Furthermore, the effectiveness of the CoS-SSI was verified experimentally with two faulty suspension faults induced into the system.
\end{abstract}

Keywords: vehicle suspension; online monitoring; CoS-SSI; nonstationary; nonlinear system 


\section{Introduction}

Modern health monitoring systems for trains are highly desirable due to the significant increase of their operational speed. As a result, the vertical and lateral contact forces between the wheel and rail are also increased significantly, especially when the suspension system is not optimized. In order to meet the needs of safety, reliability and lower operating costs of the railway vehicle, numerous investigations have been carried out by different researchers to monitor the status of the vehicle suspension system and the rail conditions [1-10], and have been reviewed recently by Alemi et al[1], Li et al[4] and Bruni et al[2] from different aspects. Based on these reviews, the condition monitoring methods for railway vehicle suspension systems can be divided into three main groups: in-depot, track-side and on-board techniques. In-depot and track-side techniques can only monitor the vehicle at selected time or locations [1, 4], while on-board methods have the potential to continuously monitor the system to provide timely information. Therefore, the onboard methods have become a main topic of research in this direction in recent years.

Li et al [5] presented an approach to detect the wheel flat using vibration in association with an Adaptive Multi-scale Morphology Filter (AMMF). The vibration signals obtained from the axle box were analysed by AMMF and the results indicated that the AMMF could be an efficient method for detecting wheel flats. Similarly, Liang et al [6] employed three commonly used timefrequency analysis techniques; Short-time Fourier Transform (STFT), Wigner-Ville Transform (WVT) and Wavelet Transform (WT), to detect wheel flat and rail surface defects by analysing axle box acceleration signals. It shows that WT has the ability to obtain good localisations both in time and frequency domains. Moreover, Mei and Ding developed a technique exploiting the dynamic interactions between different vehicle modes caused by suspension spring or damper failure[7]. In summary, all of the referred methods can be categorised as signal-based methods. However, although the signal-based methods are effective, they are difficult to apply as developing a pre-built database that includes all the possible fault conditions, e.g., various fault types and levels is very complex and difficult to implement online.

In addition to signal analysis based methods aforementioned, model-based method is also another popular technique. Wei et al [10] presented a method for the detection of vertical spring and damper faults of railway vehicle suspension, using acceleration signals only, based on the Kalman 
filter and a generalised likelihood ratio test. Moreover, Multiple Kalman filter was employed by Jesueesk et al [11] to detect and isolate the faults of the railway vehicle suspension system. Furthermore, Liu et al [12] adopted a recursive least square filter to monitor the condition of the suspension. However, an accurate dynamic model is needed for the application of model-based methods, which is often difficult to deploy for online applications.

In recent decades, Operational Modal Analysis (OMA) techniques have advanced rapidly and have been a more popular and powerful method for Structure Health Monitoring (SHM). Especially, Stochastic Subspace Identification (SSI) is regarded as one of the most popular techniques and has been developed rapidly in recent decades for SHM of the architectural structures [13, 14]. SSI method estimates a state-space model from an observed output correlation sequence by means of linear algebraic techniques; then, the modal parameters (natural frequencies, damping ratios and mode shapes) are extracted through the eigenvalue decomposition of the estimated state space model [14] and used subsequently for fault detection and diagnosis.

Compared with other OMA methods, such as Peak-Peaking (PP) and Frequency Domain Decomposition (FDD), the SSI method has the particular advantage of combining high computational robustness and efficiency when excitations (or inputs) are white noise [15]. However, its robustness will be weakened if the excitations have nonstationary contents. It is very common for the field tests to include nonstationary effect due to various reasons, such as the timevarying non-Gaussian randomness of the contacts between the wheel and rail in the real-life operation of a vehicle. To overcome this deficiency of SSI, an average correlation signal based SSI (ACS-SSI) method was proposed by Chen, et al $[16,17]$ in order to identify the dynamic characteristics of the chassis frames in a heavy truck. Their results indicated that ACS-SSI performed satisfactorily to identify modal parameters of the chassis frame using the collected nonstationary response data. Because of the effectiveness of ACS-SSI, it was employed to identify the suspension related modal parameters of a $1 / 5$ th scaled bogie roller rig. However, it was found that ACS-SSI is unable to accurately identify the targeted modal parameters on account of very severe nonstationary and nonlinearity effects.

Therefore, an improved ACS-SSI method was proposed in this paper, which takes into account the nonstationary effect to a greater extent so that it makes it more suitable for railway vehicle 
suspension monitoring. The nonstationary response is regarded primarily due to the nonstationary excitations as any real systems can be nonlinear to certain degrees due to limited linearity of spring and damping. When the amplitudes of excitations are within a certain range, the system can be more linear, otherwise the system can behave more nonlinearly. For the nonlinear operation, the responses can exhibit higher responses than that of linear one. With this understanding, the responses can be organised into a number of subsets according to the response magnitudes. The SSI procedure can then be applied to each subset to attain modal parameters, which can lead to more consistent results as the system can be locally stable for each particular amplitude range. Consequently, this subset based identification approach allows for reliable and consistent results to be obtained. Moreover, the subset is determined based on correlation signals which have better SNR, rather than on the raw responses, this method is denoted as Correlation Signal Subset based SSI (CoS-SSI), which will be depicted and evaluated comprehensively based on the data from a $1 / 5$ th scaled bogie frame on which the dynamics of the suspension system is investigated $[6,23]$.

This paper is structured with four main sections. Following this introduction, the development of the CoS-SSI algorithm is presented in Section 2. The experimental study on a $1 / 5$ th scaled bogie roller rig is detailed in Section 3. In the last section the main conclusions are drawn from this study.

\section{CoS-SSI}

Most output only system identification methods were developed based on the assumption that the system responses are stationary. However, as referred earlier, field monitored data are usually very nonstationary, such as the responses of buildings under strong winds and bridges with time-varying traffic loading [18]. In addition, the responses of the vehicle as a result of road excitations are also highly nonstationary because of the time-varying excitations due to local impacts of humps on the road and the nonlinearity of suspension systems. The identification results from output only methods will be varying as a consequence of the nonstationary response data. For instance, in the SSI scheme, the data length $N$ is limited in practice and therefore, the covariance values in the Hankel matrix may vary when the measurements contain nonstationary contents, which could result in uncertainties in the identification results [16]. Besides, the nonstationary excitations may lead to time-varying frequency contents characterised by modal components participating at 
different times [18]. It can be seen that the nonstationary problem is a challenging issue and therefore a lot of investigations have been conducted by different scholars [19]-[21].

Lin and Chiang et al $[19,20]$ addressed the nonstationary problem by adopting the correlation technique. A theoretical justification was given in reference [19] and stated that the nonstationary correlation functions evaluated at an arbitrary, fixed time instants of structural response have the same form as free decay of the structure with certain initial conditions. Their theory was developed based on the principle [21] that the cross-correlation functions of two stationary processes can be expressed as free impulse responses. In summary, the theory developed in [19] indicated that the nonstationary problem could be reduced to a stationary problem if the nonstationary correlation functions are evaluated at a fixed time instant.

Based on this concept, a method based on the correlation signals combined with the framework of SSI, denoted as ACS-SSI, was proposed by Chen et al $[16,17]$. The key steps to obtain average correlation signals are shown as follows $[16,17]$ and the framework of SSI can be found in $[13$, 22]:

1) Collect $K$ numbers of data segments from the measurements of $l$ channels; this can be achieved by using multiple records or by segregating a long record into small ones.

2) Calculate the cross-correlation signals of each segment between $l$ channels:

$$
r^{i p}(m)=\frac{1}{N} \sum_{n=0}^{N-1-m} y^{p}(n) y^{i}(n+m)
$$

Where $N$ is the length of each segment; $i=1,2, \ldots, l$ is the channel number; $p$ is the reference channel; $n$ is the time sequence and $m$ is the delayed time. In order to improve the calculation efficiency, the Fast Fourier Transform (FFT) algorithm is applied to obtain the correlation signals.

3) Averaging the correlation signals from different segments to obtain the average correlation signals for the corresponding channels.

$$
\overline{r^{i p}(m)}=\frac{1}{K} \sum_{k=1}^{K} r_{k}^{i p}(m)
$$


Where $K$ is the number of segments.

The ACS-SSI suppresses the high noise and nonstationary signals by calculating the correlation signals and averaging them before applying in SSI method for modal identification. As referred previously, it has been proved in $[16,17]$ that ACS-SSI has excellent ability to identify the modal parameters of the truck frame effectively with only the output responses when the truck runs on the constructed road.

However, because of the strong nonstationary characteristics in response signals, the amplitudes of correlation signal can spread within a very wide dynamic range. This means that the ensemble average in ACS-SSI will take less account of the correlation signals with very low amplitudes. Especially, low amplitude correlation signals often contain information associated with the vibration modes with higher damping coefficients and thus the vibration modes are less frequently excited. This means that averaging over a full set of data may lead to inadequate identification results in that modes with high damping and cannot be identified reliably. Moreover, because of the inevitable effects of system nonlinearity, different amplitudes of correlations signals may come from different regimes of a nonlinear system. In other words, the higher vibration amplitudes may indicate that the system operates with a set of modal parameters which can be slightly different from that of those with lower amplitudes. As a consequence, ACS-SSI can identify inconsistent and less repeatable results, which probably change with magnitudes of measured responses due to the nonlinear effect.

Based on the above analysis, a CoS-SSI method can be proposed to overcome the deficiency and limitations in applying ACS-SSI to nonstationary and quasi-nonlinear scenarios. In the CoS-SSI method, the correlation signals are divided into several subsets according to their magnitudes and then averaging the segments of correlation signals with respect to each subset, rather than conducting the averaging step to the full segments of correlation signals. The averaged correlation signals obtained from these subsets are separately applied in SSI to identify the modal parameters.

In this study, the signal magnitude is presented by its root mean square (RMS) value. Hence, the first step is to calculate RMS values of all correlation segments and to develop a RMS matrix. For example, as shown in Figure 1, if the raw signals have $l$ channels and the signals from each channel are divided into $K$ segments, the number of correlation signal segments obtained from 
each segment is $l \times l$ and therefore can obtain $K \times l^{2}$ correlation signal segments in total. Each segment of the correlation signals can obtain a RMS value, consequently, a RMS value matrix in size of $K \times l^{2}$ can be developed. The second step is to select the minimum RMS value from each row, allowing for a vector $\mathbf{R}_{\mathrm{m}}$ to be obtained which contains $K$ elements. The third step is to obtain the maximum and minimum values from vector $\mathbf{R}_{\mathrm{m}}$. The fourth step is to calculate the intervals using the maximum values to subtract the minimum values which are obtained in the previous step and then divide the subsets number $J$. The final step is to categorise the correlation segments into different subsets by locating the RMS values obtained in the first step at different intervals. The value of the subset number is always chosen 3 or 4 considering the calculation efficiency and the accuracy of the identification results.

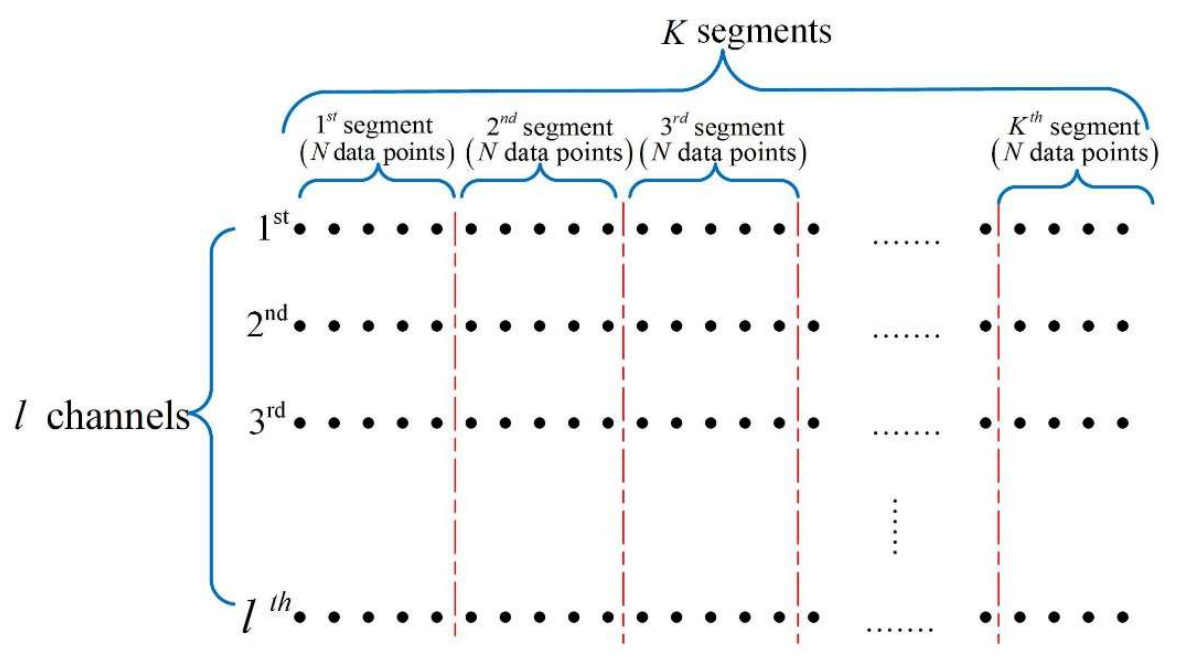

Figure 1 Schematic of raw signal segregation

This unique scheme of implementing ACS-SSI to a series of subset correlation signals with sequentially changed (increases or decreases) magnitudes allows the system nonlinearity to be suppressed greatly. For clarity, the CoS-SSI method is further summarised within the flow chart as shown in Figure 2. It highlights the improved steps (in red box) made in this study to suppress the effect of nonlinearity and nonstationary. 


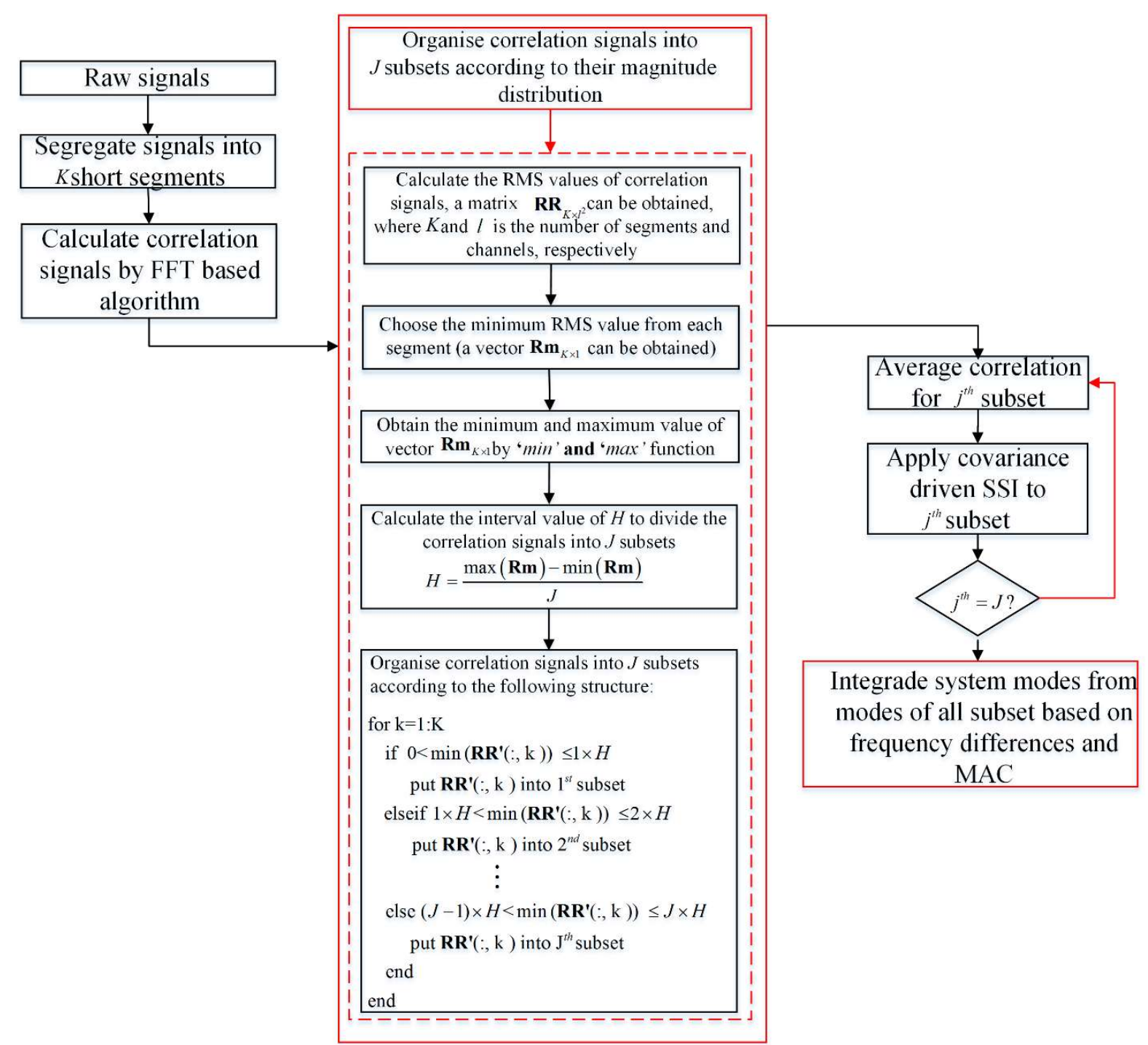

Figure 2 Flow chart of CoS-SSI

In addition, it is well known that the Stabilisation Diagram (SD) is a powerful tool to filter out the false modes in OMA $[13,14]$. The principle of SD is that the true modes of system are stable irrespective of the number of rows (order) in Hankel matrix, but the false modes are not. Therefore, a threshold is set up to confirm the consistency of the modal parameters identified from two conjunction Hankel orders. The threshold includes $\varepsilon_{f}, \varepsilon_{\xi}, \varepsilon_{M A C}$, given in equations(3), (4) and (5).The real modes have to simultaneously satisfy the three equations.

$$
\frac{f_{i}-f_{i-1}}{f_{i}} \times 100 \%<\varepsilon_{f}
$$




$$
\begin{aligned}
& \frac{\xi_{i}-\xi_{i-1}}{\xi_{i}} \times 100 \%<\varepsilon_{\xi} \\
& 1-M A C(i: i-1)<\varepsilon_{M A C} \\
& \operatorname{MAC}(i: i-1)=\frac{\left|\left(\phi_{i}\right)^{T} \phi_{i}\right|^{2}}{\left(\phi_{i}\right)^{T}\left(\phi_{i}\right)\left(\phi_{i-1}\right)^{T} \phi_{i-1}}
\end{aligned}
$$

Where MAC is the acronym of Modal Assurance Criterion; $f_{i}, \xi_{i}, \phi_{i}$ are the identified frequency, damping ratio and mode shape when the Hankel matrix order is $i$. The values of $\varepsilon_{f}, \varepsilon_{\xi}, \varepsilon_{M A C}$ were set at $0.1,0.2$ and 0.5 , respectively.

In this paper, a second threshold $\delta$ was set up by calculating the rate of the stable points over the maximum calculated order (row) of the Hankel matrix to filter out the false modes in a further step, shown in equation (6):

$$
\frac{x}{y} \times 100 \% \geq \delta
$$

Where $x$ is the number of stable points identified for an exact frequency; $y$ is the maximum order of Hankel matrix in the identification process.

\section{Online modal identification of scaled bogie}

\subsection{Theoretical modal parameters}

\section{a) Test rig introduction}

Bogie is an essential part in the railway vehicle suspension system which is connected to the wheelsets through the primary suspension system and connected to the car body through the secondary suspension system. The 1/5th scaled bogie installed on the roller rig, which was employed frequently to study the dynamics of railway vehicles $[6,23]$, was used in this study to investigate the performance of the proposed method.

As shown in Figure 3(a), the main parts of this roller rig include two wheelsets and a bogie frame. Eight mount bushings, a pair at each corner, are considered as the primary suspension to connect the bogie frame and the wheelsets. In addition, a control panel is equipped which can be used to adjust the running speed of the roller continuously. Furthermore, a frame and two joints are 
employed to constrain the movement of the bogie in longitudinal direction. However, this roller rig was designed to investigate the railway vehicle lateral dynamics, therefore, the vertical dynamical characteristics were not fully considered. The bounce and pitch modes were extremely close, which was untrue for the real vehicle vertical dynamics. It is well known that the close modes are difficult to identify correctly, even the SSI method is much superior than most of the other methods in this aspect. Therefore, two weights $(2 \mathrm{~kg} / \mathrm{each})$ are attached on the centreline of the bogie frame to separate the bounce and pitch modes.

For the purpose of generating a relative real rail excitation, the wheel profiles were machined as scale versions of BR P8 and the rollers were machined as scale of BS110 with a rail profile without the rail cant[23]. The rollers were driven by a motor through a belt. A schematic side view of the roller rig system is presented in Figure 3(b).
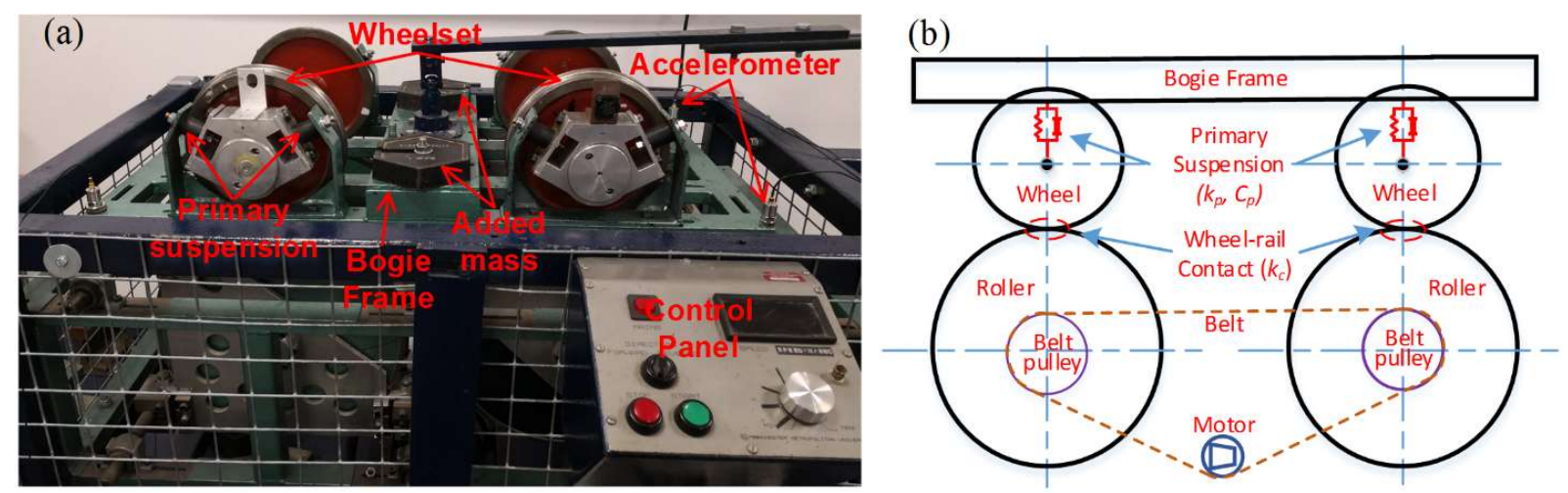

Figure 3 1/5th scaled roller rig and side view schematic

\section{b) 7-DOF mathematical model}

A 7-DOF linear model of the experimental scaled roller rig was developed in this section for the purpose of obtaining the theoretical modal parameters. The 7-DOF model included bounce, pitch and rolling movements of the bogie frame and the bounce movement of the wheels. The schematic of the 7-DOF model is shown in Figure 4. 


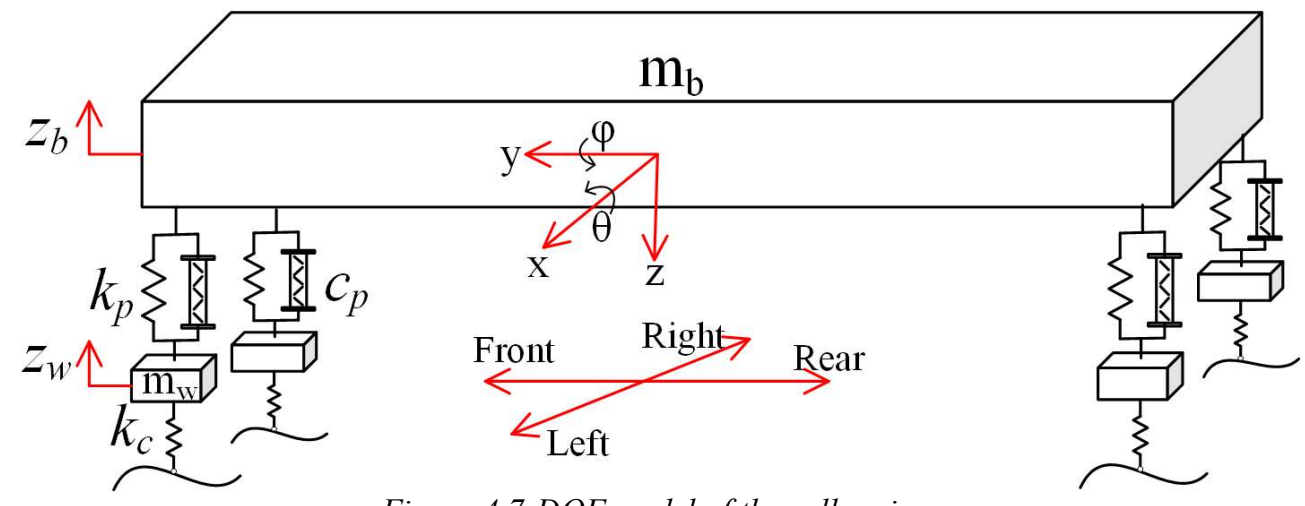

Figure 4 7-DOF model of the roller rig

The kinematic equations can be developed according to the Newton's second law, shown in equation (7)-(14):

Bounce of the bogie frame:

$$
\begin{aligned}
m_{b} \ddot{z}= & -k_{p f l}\left(z_{b f l}-z_{w f l}\right)-k_{p f r}\left(z_{b f r}-z_{w f r}\right)-k_{p r l}\left(z_{b r l}-z_{w r l}\right)-k_{p r r}\left(z_{b r r}-z_{w r r}\right) \\
& -c_{p f l}\left(\dot{z}_{b f l}-\dot{z}_{w f l}\right)-c_{p f r}\left(\dot{z}_{b f r}-\dot{z}_{w f r}\right)-c_{p r l}\left(\dot{z}_{b r l}-\dot{z}_{w r l}\right)-c_{p r r}\left(\dot{z}_{b r r}-\dot{z}_{w r r}\right)
\end{aligned}
$$

Pitch of the bogie frame:

$$
\begin{aligned}
I_{p} \ddot{\theta}= & -a\left[k_{p f l}\left(z_{b f l}-z_{w f l}\right)+k_{p f r}\left(z_{b f r}-z_{w f r}\right)+c_{p f l}\left(\dot{z}_{b f l}-\dot{z}_{w f l}\right)+c_{p f r}\left(\dot{z}_{b f r}-\dot{z}_{w f r}\right)\right] \\
& +a\left[k_{p r l}\left(z_{b r l}-z_{w r l}\right)+k_{p r r}\left(z_{b r r}-z_{w r r}\right)+c_{p r l}\left(\dot{z}_{b r l}-\dot{z}_{w r l}\right)+c_{p r r}\left(\dot{z}_{b r r}-\dot{z}_{w r r}\right)\right]
\end{aligned}
$$

Roll of the bogie frame:

$$
\begin{aligned}
I_{R} \ddot{\varphi}= & -b\left[k_{p f l}\left(z_{b f l}-z_{w f l}\right)+k_{p r l}\left(z_{b r l}-z_{w r l}\right)+c_{p f l}\left(\dot{z}_{b f l}-\dot{z}_{w f l}\right)+c_{p r l}\left(\dot{z}_{b r l}-\dot{z}_{w r l}\right)\right] \\
& +b\left[k_{p f r}\left(z_{b f r}-z_{w f r}\right)+k_{p r r}\left(z_{b r r}-z_{w r r}\right)+c_{p f r}\left(\dot{z}_{b f r}-\dot{z}_{w f r}\right)+c_{p r r}\left(\dot{z}_{b r r}-\dot{z}_{w r r}\right)\right]
\end{aligned}
$$

Bounce of the four wheels:

$$
\begin{aligned}
& m_{w f l} \ddot{z}_{w f l}=k_{p f l}\left(z_{b f l}-z_{w f l}\right)+c_{p f l}\left(\dot{z}_{b f l}-\dot{z}_{w f l}\right)-k_{c f l}\left(z_{w f l}-z_{r f l}\right) \\
& m_{w f r} \ddot{z}_{w f r}=k_{p f r}\left(z_{b f r}-z_{w f r}\right)+c_{p f r}\left(\dot{z}_{b f r}-\dot{z}_{w f r}\right)-k_{c f r}\left(z_{w f r}-z_{r f r}\right) \\
& m_{w r l} \ddot{z}_{w r l}=k_{p r l}\left(z_{b r l}-z_{w r l}\right)+c_{p r l}\left(\dot{z}_{b r l}-\dot{z}_{w r l}\right)-k_{c r l}\left(z_{w r l}-z_{r r l}\right) \\
& m_{w r r} \ddot{z}_{w r r}=k_{p r r}\left(z_{b r r}-z_{w r r}\right)+c_{p r r}\left(\dot{z}_{b r r}-\dot{z}_{w r r}\right)-k_{c r r}\left(z_{w r r}-z_{r r r}\right)
\end{aligned}
$$

Where $z_{b i}$ is vertical displacement of the bogie frame; $\theta:$ pitch angle of the bogie frame; $\varphi$ : roll angle of the bogie frame; $z_{w i}$ : vertical displacement of the wheel caused by track irregularity and 
wheel faults. ( $i=f l, f r, r l, r r$ meaning position of front-left, front-right, rear-left and rear-right). The other symbols are detailed in Table 1.

The relationships between the displacements at the four corners of the bogie frame and the centre gravity are given as follows:

$$
\begin{aligned}
& z_{b f l}=z_{b}+a \theta+b \varphi, \quad z_{b f r}=z_{b}+a \theta-b \varphi \\
& z_{b r l}=z_{b}-a \theta+b \varphi, z_{b r r}=z_{b}-a \theta-b \varphi
\end{aligned} ;
$$

These equations can be reformulated into state space form and therefore the modal parameters can be extracted from the state matrix. The physical parameters of the roller rig were measured and given in Table 1. Particularly, $I_{p}$ and $I_{R}$ were obtained through developing a $3 \mathrm{D}$ model in the SOLIDWORKS. Moreover, the suspension stiffness of the bogie frame is hard to measure directly, therefore, it was approximated by the relationship of the installation angle $(\theta)$, shown in Figure 5(b). Firstly, the stud mount was tested on an INSTRON 3369 universal testing system, which will be introduced in detail in Section 3.4. The tested result of the force-displacement curve was presented in Figure 5(a). Secondly, the vertical stiffness of the stud mount was calculated when the compressive extension was around $1 \mathrm{~mm}$ considering the load ability of the employed stud mounts and the weight of the bogie frame. It can be seen that the force is around $113 \mathrm{~N}$ when the compressive extension is $1 \mathrm{~mm}$; yet the stiffness is $113 / 0.001=1.13 e 5 \mathrm{~N} / \mathrm{m}$. The installation angle $\theta$ of the stud mount is around $\pi / 3$, therefore the suspension stiffness is around $2 \times 1.13 e 5 \times \cos (\pi / 3)=1.13 e 5 \mathrm{~N} / \mathrm{m}$. In addition, the damping of the suspension and the contact stiffness between wheel and rail are chosen according to the reference [6].
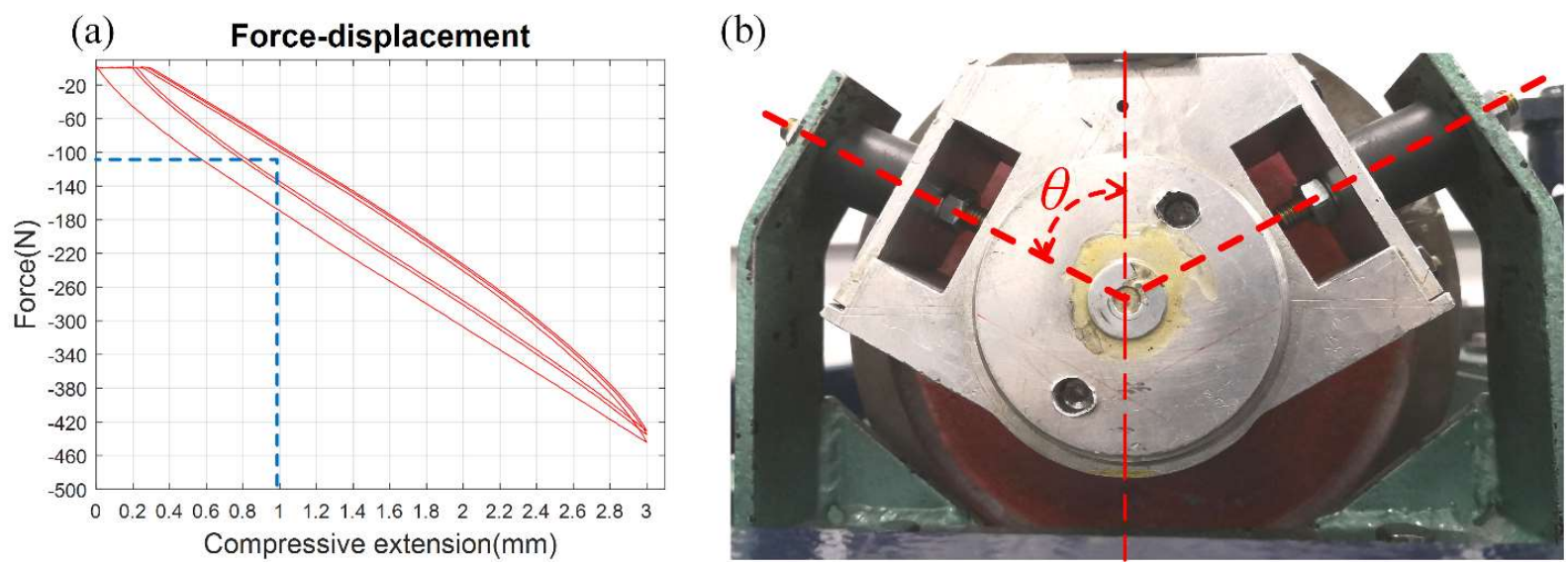
Figure 5 Stud mount force-displacement and installation direction

Table 1 Physical parameters of the roller rig

\begin{tabular}{crr}
\hline Symbol & Meaning & Value \\
\hline$m_{b}$ & Mass of the bogie frame & $30.95 \mathrm{~kg}$ \\
$m_{w}$ & Half Mass of the wheelset & $12.5 / 2 \mathrm{~kg}$ \\
$I_{p}$ & Bogie frame pitch inertia & $1.23 \mathrm{~kg} \bullet \mathrm{m}^{2}$ \\
$I_{R}$ & Bogie frame roll inertia & $0.67 \mathrm{~kg} \bullet \mathrm{m}^{2}$ \\
$k_{p f l}, k_{p f r}, k_{p r l}, k_{p r r}$ & Stiffness of the primary suspension & $1.13 \times 10^{5} \mathrm{~N} / \mathrm{m}$ \\
$c_{p f l}, c_{p f r}, c_{p r l}, c_{p r r}$ & Damping of the primary suspension & $133.5 \mathrm{Ns} / \mathrm{m}$ \\
$k_{c f l}, k_{c f r}, k_{c r l}, k_{c r r}$ & Contact stiffness between wheel and track & $8.93 \times 10^{6} \mathrm{~N} / \mathrm{m}$ \\
$a$ & Half of the wheelbase & $0.42 / 2 \mathrm{~m}$ \\
$b$ & Half of the gauge & $0.39 / 2 \mathrm{~m}$ \\
\hline
\end{tabular}

\section{c) Theoretical modal parameters}

The theoretical modal parameters were obtained by substituting the parameters (tabulated in Table 1) into the developed model. In this paper, only the rigid modes of the bogie frame, related to the primary suspension, were considered. They are bounce, pitch and roll modes, shown in Figure 6. The resonance frequencies of the three modes are $19.15 \mathrm{~Hz}, 20.18 \mathrm{~Hz}$ and $25.40 \mathrm{~Hz}$, respectively; the corresponding damping ratios are $6.99 \%, 7.37 \%$ and $9.28 \%$.
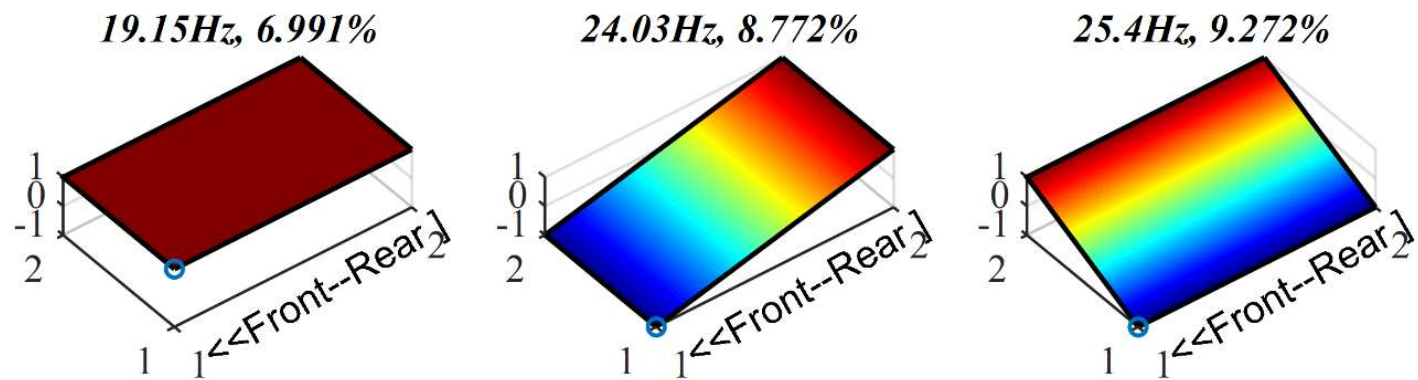

Figure 6 Theoretical modal parameters of roller rig added with $4 \mathrm{~kg}$ mass

At the same time, in order to confirm whether the flexible modes of the bogie frame have effects on the rigid modes or not, a finite element model (FEM) of the bogie frame was constructed in ANSYS/Workbench software to investigate its flexible modes. The model was in full-scale and it was meshed into 9828 elements with 21124 nodes. The first four flexible modes obtained from the FEM were presented in Figure 7. It can be seen that the resonant frequency of the first flexible bending mode is around $86.43 \mathrm{~Hz}$, which is nearly three times higher than the rigid roll mode of 
the bogie frame. Therefore, it can be recognised that the flexible modes have limited effects on the suspension related rigid modes of the bogie frame, which means the developed rigid model is reasonable to analyse the dynamic characteristics of the suspension system.

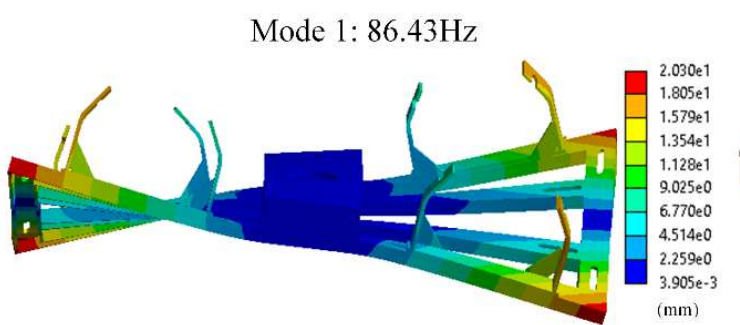

Mode 3: $142.38 \mathrm{~Hz}$

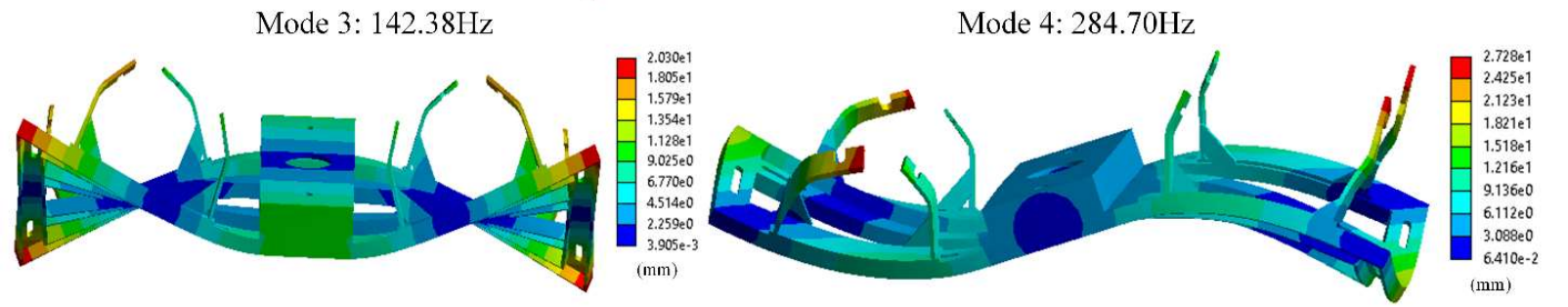

Figure 7 Flexible modes of bogie frame

\subsection{Raw signal characteristics}

In the experiment, four accelerometers were mounted on the four corners of the bogie frame, shown in Figure 3(a), to collect the responses caused by the irregularities of the roller (rail) and the wheel. As shown in Figure 8, the accelerometer is CA-YD-185 from SINOCERA. This accelerometer is used widely due to its high sensitivity $\left(50 \mathrm{mV} / \mathrm{m} / \mathrm{s}^{2}\right)$ and wide frequency band (from $0.5 \mathrm{~Hz}$ to $5000 \mathrm{~Hz}$ ) along with small mass. A YE6231 four channel data acquisition system with a laptop were used to collect the data. The system can acquire the vibration data at $96 \mathrm{kHz}$ at a data accuracy of 24bit, which ensures great accuracy in capturing both the high and low amplitude vibration in the low frequency range for suspension analysis. 
(a) Accelerometers (CA-YD-185)

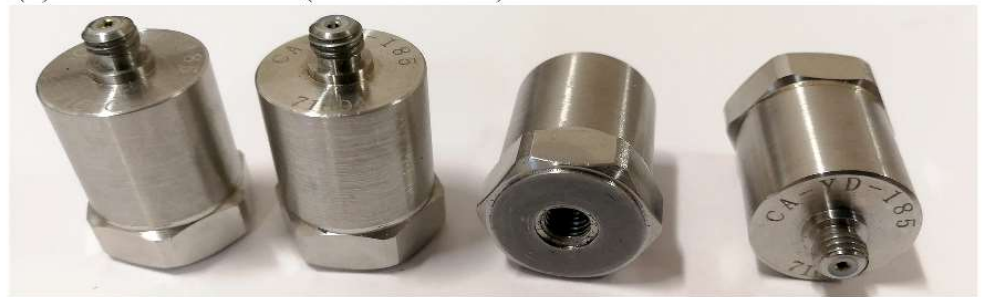

(b) Data Acquisition(YE6231)

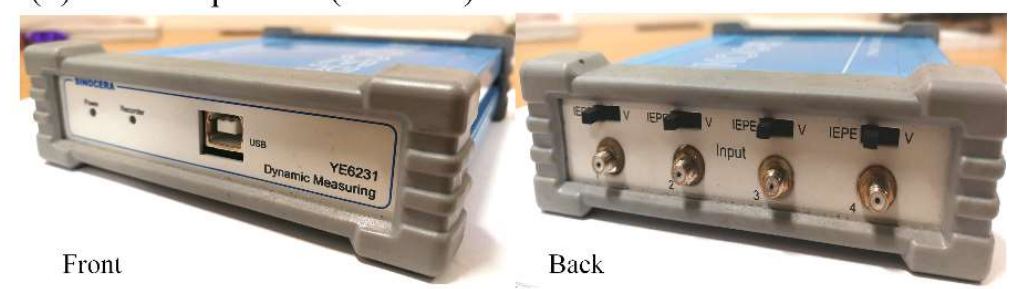

Figure 8 Accelerometers and data acquisition system

The sampling rate of YE6231 was set at $1500 \mathrm{~Hz}$ for the experiments. It is apparent that the sampling frequency is much higher than the requirement of Nyquist sampling theory. This is to confirm the reliability of the CoS-SSI method since this is a primary study. However, the sampling rate can be lower in real application for the scope of improving calculation efficiency. Generally, 5 times the maximum interested natural frequency would be enough. In order to confirm the reliability of the CoS-SSI further, the data was collected under varying speeds, which presented a more nonstationary scenario, but can better illustrate the real operations of railway vehicles with frequent acceleration and deceleration.

The sampling length was 40s for each test and the test was repeated 10 times under the same condition. An example of the raw signals in the time domain is shown in Figure 9. It can be seen that the amplitudes of the responses are changing significantly with time, which indicate that the responses are very nonstationary. The power spectrum densities (PSD) corresponding to each channel are presented below the time-domain signals. The two spectral peaks can be observed at $20 \mathrm{~Hz}$ and $26 \mathrm{~Hz}$ respectively. They are similar to the calculated frequencies of the pitch and rolling modes shown in Figure 6, showing that the spectrum allows the modal frequency to be estimated approximately. However, it is difficult to find other modal parameters based on the spectrum analysis. Particularly spectral amplitudes at the four corners are significantly different. Such differences have little similarities to the modal shapes in Figure 6, and therefore provide limited information for estimating the modal shapes. Instead, the spectral differences are caused by compound effects including modes coupling due to the closeness of $19.15 \mathrm{~Hz}$ and $20.18 \mathrm{~Hz}$, and 
various noises including system nonlinearity, asymmetric excitations between the corners and measurement deviations. This also means that OMA methods are needed to minimise these noises and achieve accurate system identification.
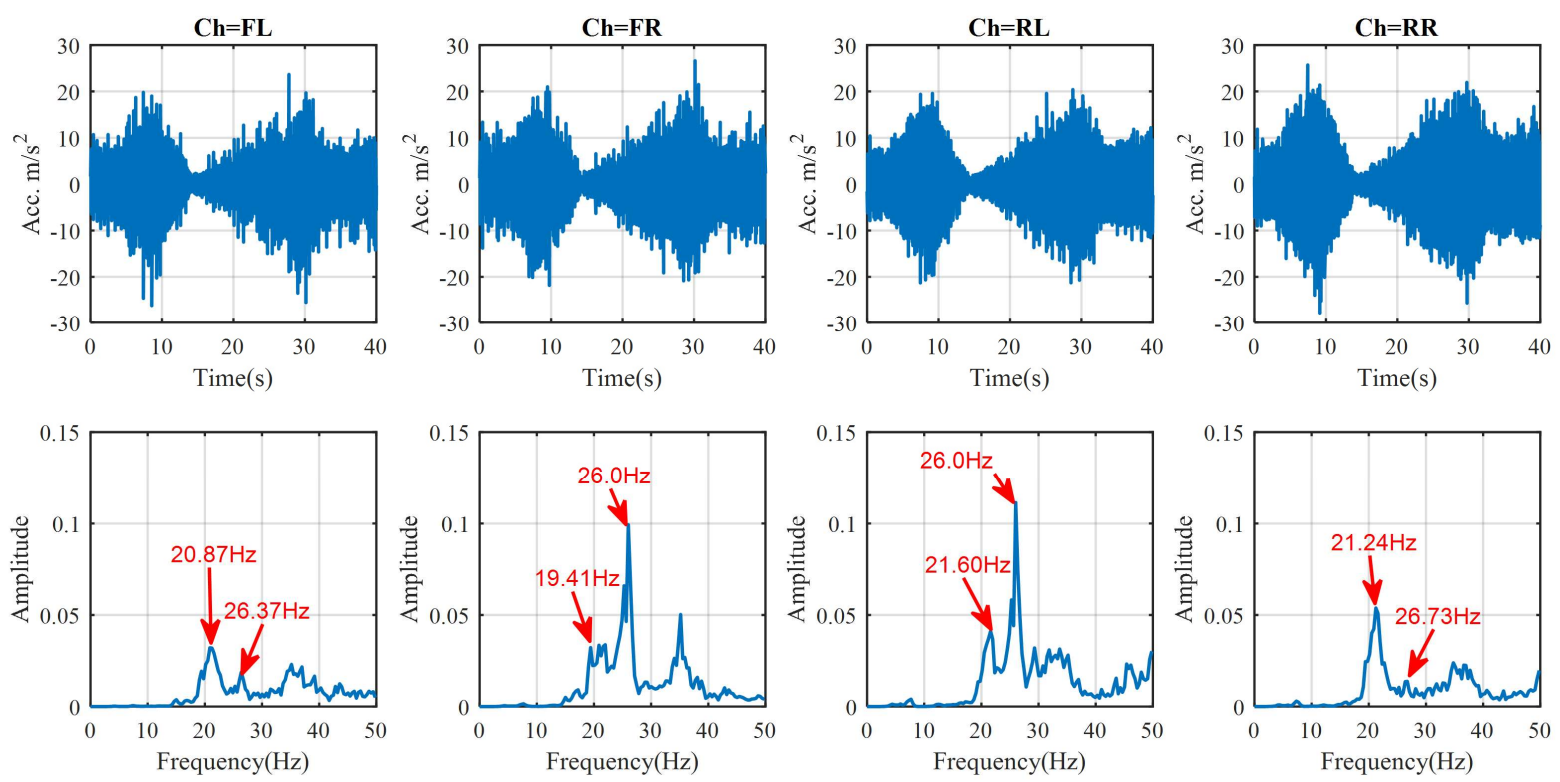

Figure 9 Raw signals and PSD (FL: front left; FR: front-right; RL: rear-left; RR: rear-right)

\subsection{Modal identification results and discussion}

To evaluate the performance comprehensively, the modal parameters identified by COS-SSI are compared with that of Cov-SSI and ACS-SSI when they are all applied to the same data from the $1 / 5^{\text {th }}$ scaled bogie. As the sampling frequency was $1,500 \mathrm{~Hz}$, the data length has 60,000 points for 40 seconds and they were divided into 14 segments, each having $N=4096$ points for each segment, which covers more than 50 periods of the lowest mode of interest and allows for significant noise reduction. Then, the correlation signals between four channels were calculated for each of the segments, yielding $K=140$ segments of correlation signals in total for the ensample average to reduce noise further.

SD and the rate of stable point $\operatorname{rate}(\delta)$ were adopted to filter out the spurious modes. Firstly, the SD identified by Cov-SSI method are presented in Figure 10(a). It can be seen that two relative stable modes were presented in the SD, which are around $20 \mathrm{~Hz}$ and $21 \mathrm{~Hz}$. Then, the rate of stable points in the SD are presented in Figure 10(b). The two relative stable modes were selected when the second threshold $(\delta)$ was set at 0.6. The selected modes are presented in Figure 11(a). 
Moreover, the MAC referenced with theoretical mode shapes are presented in Figure 11(b). It can be found that the identified modes are bounce and pitch respectively. The relative errors of the identified bounce and pitch frequencies are $4.28 \%$ and $4.66 \%$, compared with the theoretical one, which is in the acceptable range. However, the roll mode has not been identified at all, showing that Cov-SSI is not able to suppress the noise sufficiently to resolve the roll mode.
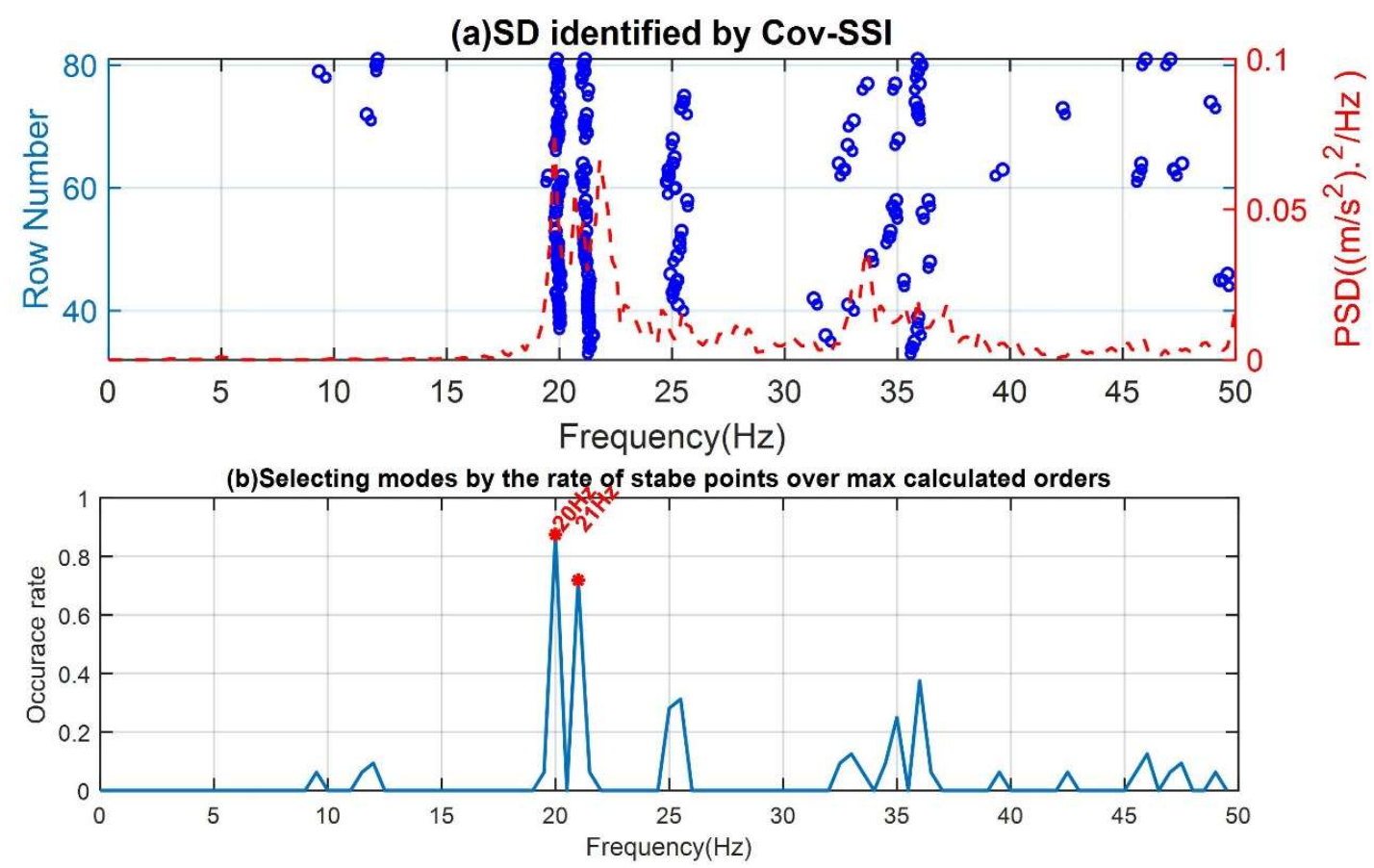

Figure 10 (a)SD identified by Cov-SSI and (b) rate of stable points

(a)

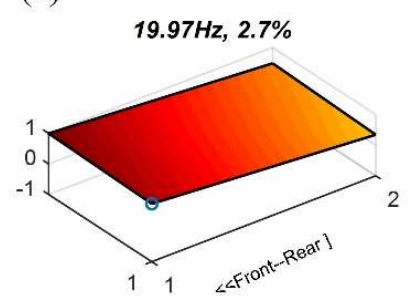

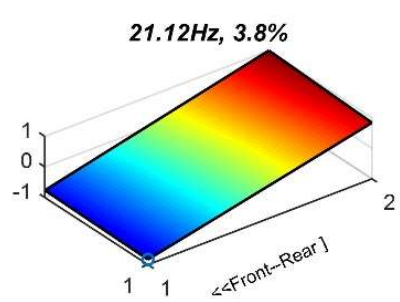

(b)

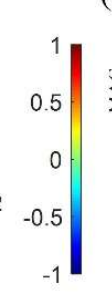

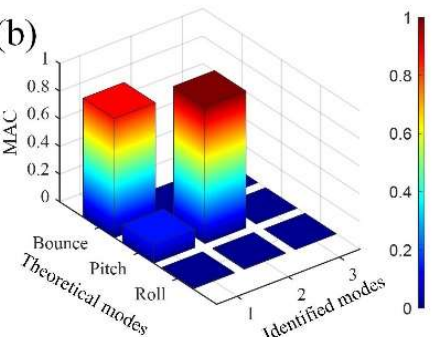

Figure 11 (a)Cov-SSI identified results and (b) MAC values compared with theoretical modes

The results obtained by ACS-SSI are presented in Figure 12 and Figure 13, which are obtained by . the same thresholds as that of Cov-SSI. This method allows the pitch and roll modes to be identified but not the bounce mode. It is worthy to note that the bounce mode could be identified if the second threshold was set at 0.3 according to the results presented in Figure 12(b). However, this low threshold could result in two false modes around $10 \mathrm{~Hz}$ and $36 \mathrm{~Hz}$. This means that the 
threshold values should be sufficiently higher to exclude the false modes to achieve a more reliable result.

(a)SD identified by ACS-SSI

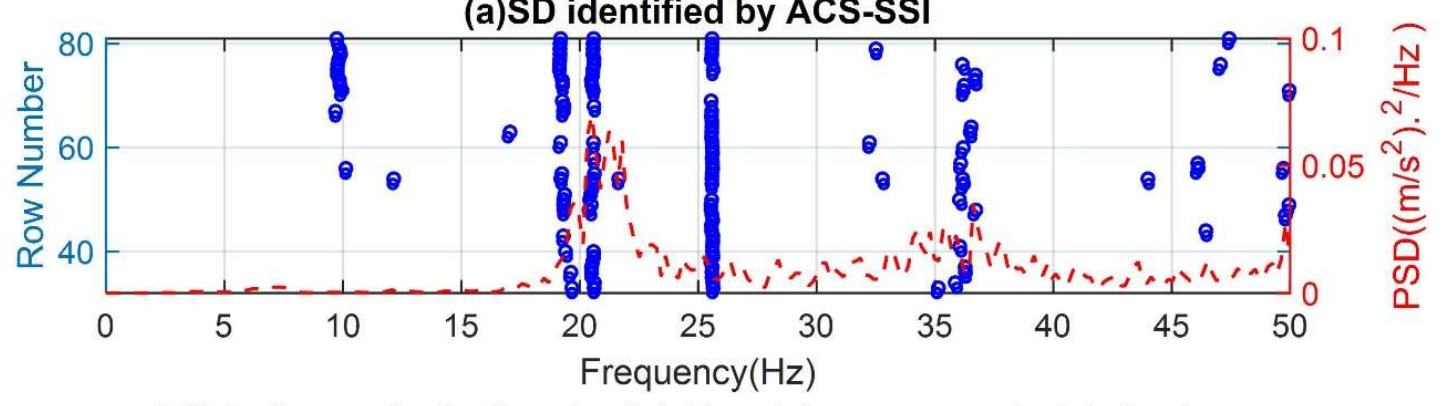

(b)Selecting modes by the rate of stable points over max calculated orders

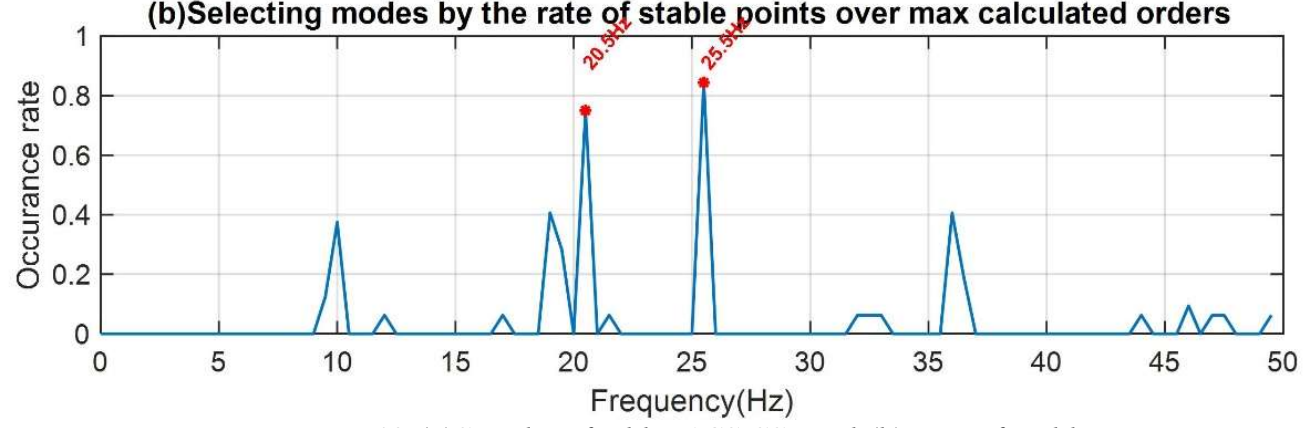

Figure 12 (a)SD identified by ACS-SSI and (b) rate of stable rates

(a)

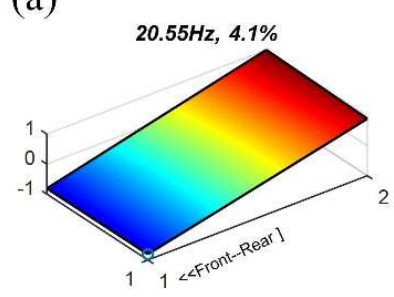

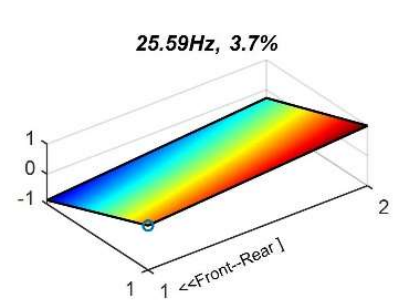

(b)

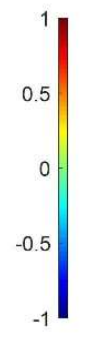

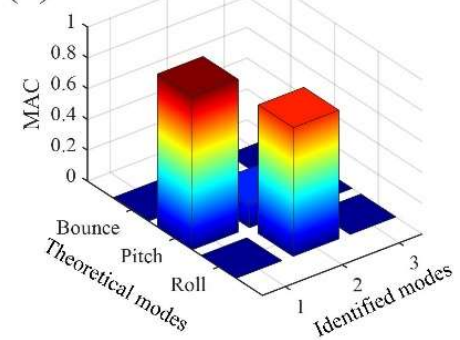

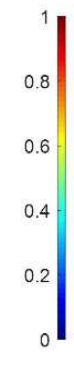

Figure 13 (a) Modes identified by ACS-SSI, and (b) MAC values compared with theoretical modes

As mentioned earlier, the ACS-SSI method averages the ensemble of correlation signals which means that correlation signals with small amplitudes contribute less to the final results. The small amplitude correlation signals are often related to modes with higher damping and lower excitations. Therefore, in the CoS-SSI, the 140 correlation signal segments were categorised into 3 subsets based on their amplitudes. The subset number was selected on account of identification accuracy and calculation efficiency.

The SDs identified by CoS-SSI method respective to each subset are presented in Figure 14(a $\left.\mathrm{a}_{1}\right)$, $\left(b_{1}\right)$ and $\left(c_{1}\right)$; the figures showing the rate of stable points are presented below them, as shown in 
Figure $14\left(a_{2}\right),\left(b_{2}\right)$ and $\left(c_{2}\right)$. It can be observed that the SDs are much stable than the results identified by Cov-SSI and ACS-SSI. Consequently, the second threshold was set at a higher value, set at 0.8 . This means that the results identified by the new method will be more reliable.

The results identified from the three subsets by CoS-SSI are presented in Figure $15\left(a_{1}\right),\left(b_{1}\right)$ and $\left(c_{1}\right)$, respectively, and the corresponding MAC values compared with theoretical mode shapes are presented below. It can be observed from Figure $15\left(\mathrm{a}_{1}\right)$ and $\left(\mathrm{a}_{2}\right)$ that the bounce, pitch and roll modes are identified from the first subset and were largely in agreement with the theoretical results. In addition, it can also be seen that bounce and pitch modes are identified in the second subset; pitch and roll modes are identified in the third subset. In summary, all suspension related bogie frame rigid modes have been identified by the CoS-SSI method. Although it is noticeable that the identified modes are not exactly identical to the theoretical modes, the errors are acceptable considering the limits of the model developing process. Furthermore, it is also evident that the frequencies of the same mode identified in each subset is different, but the differences are quite small. It is reasonable to assume that the small difference is caused by the nonlinearity of the mount bushing.

$\left(a_{1}\right)$ SD identified by CoS-SSI ( $j=1 / 1$ st subset)

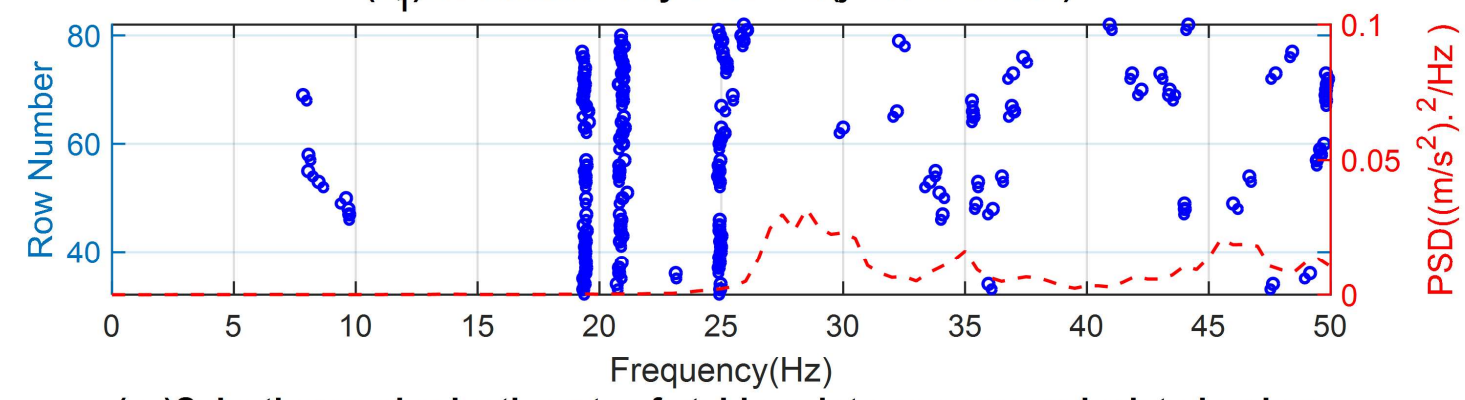

$\left(a_{2}\right)$ Selecting modes by the rate of stable points over max calculated orders

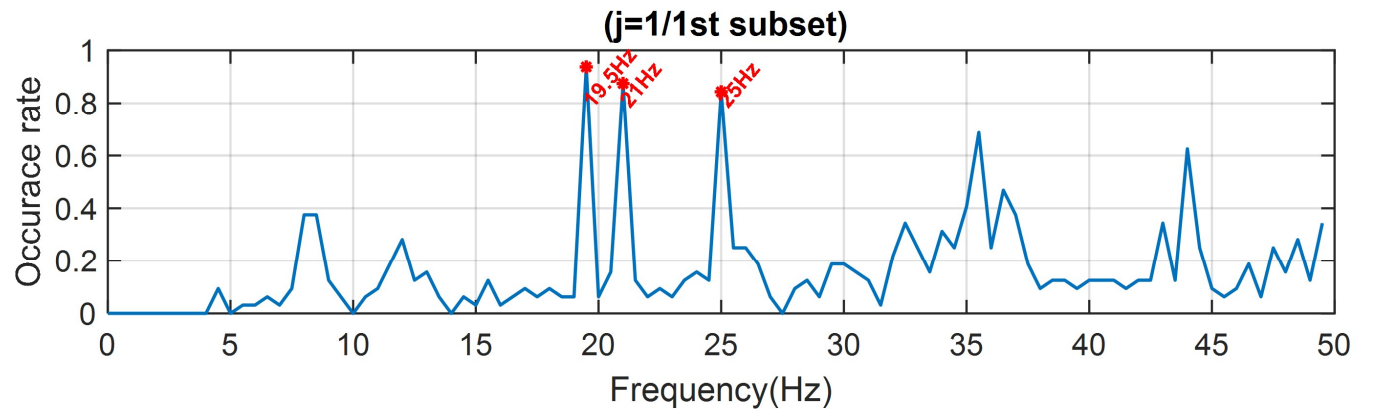


$\left(b_{1}\right) S D$ identified by CoS-SSI ( $\mathrm{j}=2 / 2 \mathrm{nd}$ subset)

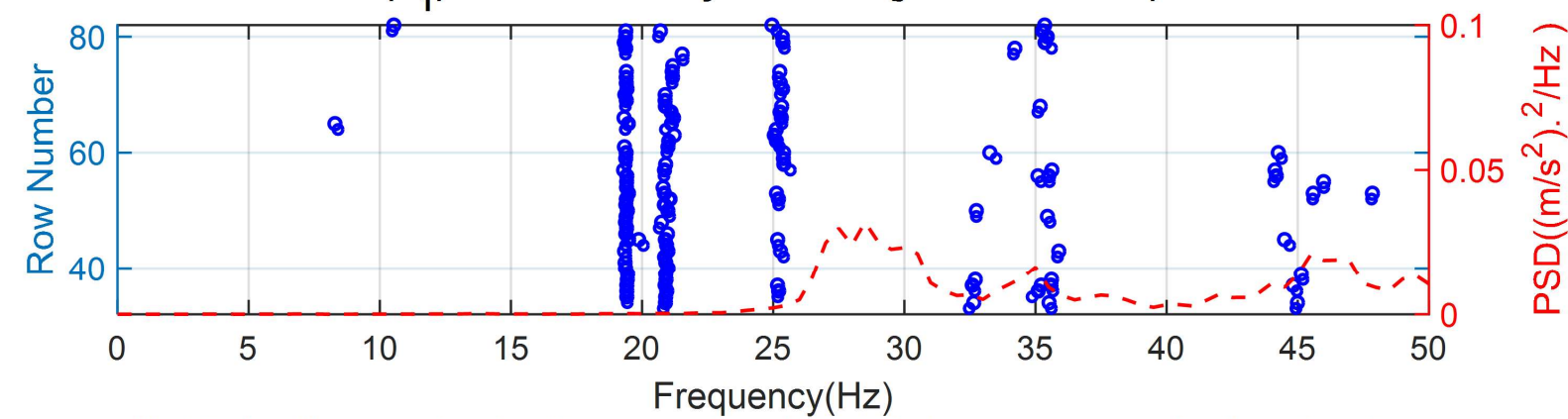

$\left(b_{2}\right)$ Selecting modes by the rate of stable points over max calculated orders

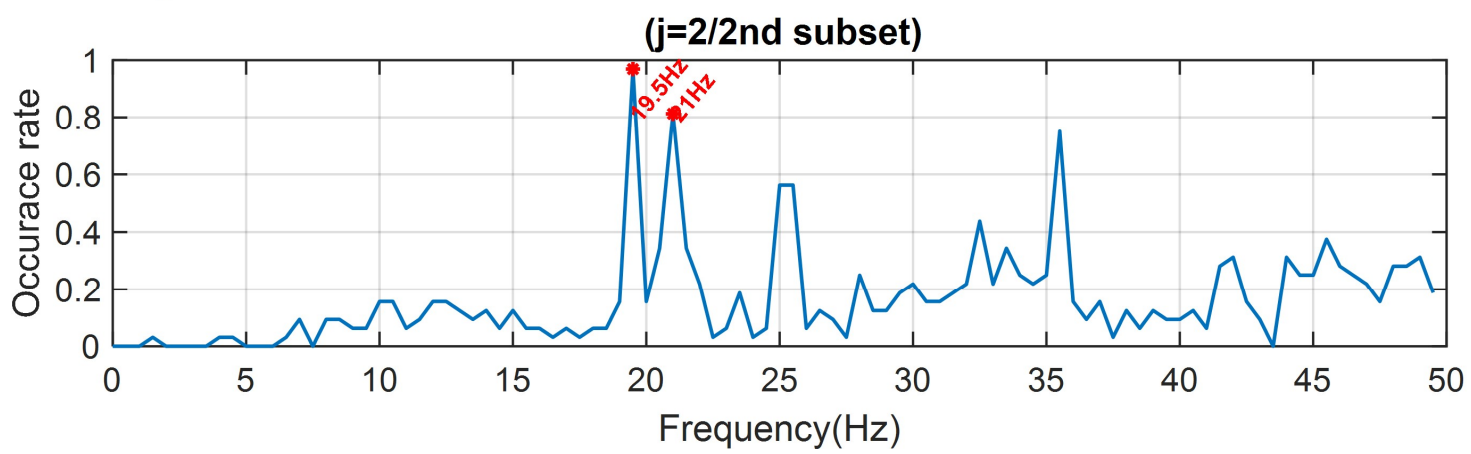

$\left(c_{1}\right)$ SD identified by CoS-SSI ( $j=3 / 3$ rd subset)

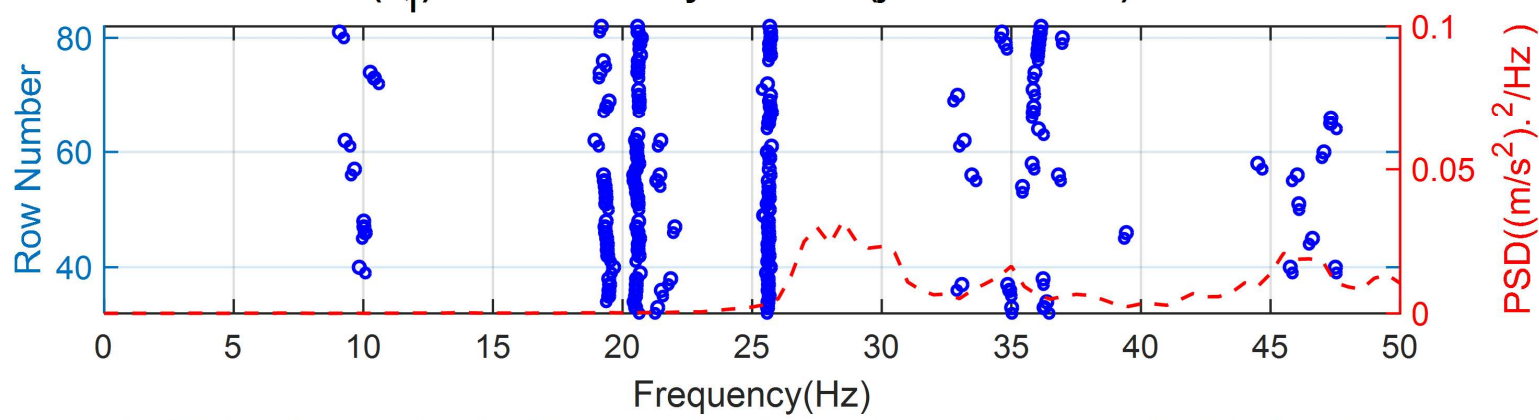

$\left(c_{2}\right)$ Selecting modes by the rate of stable points over max calculated orders

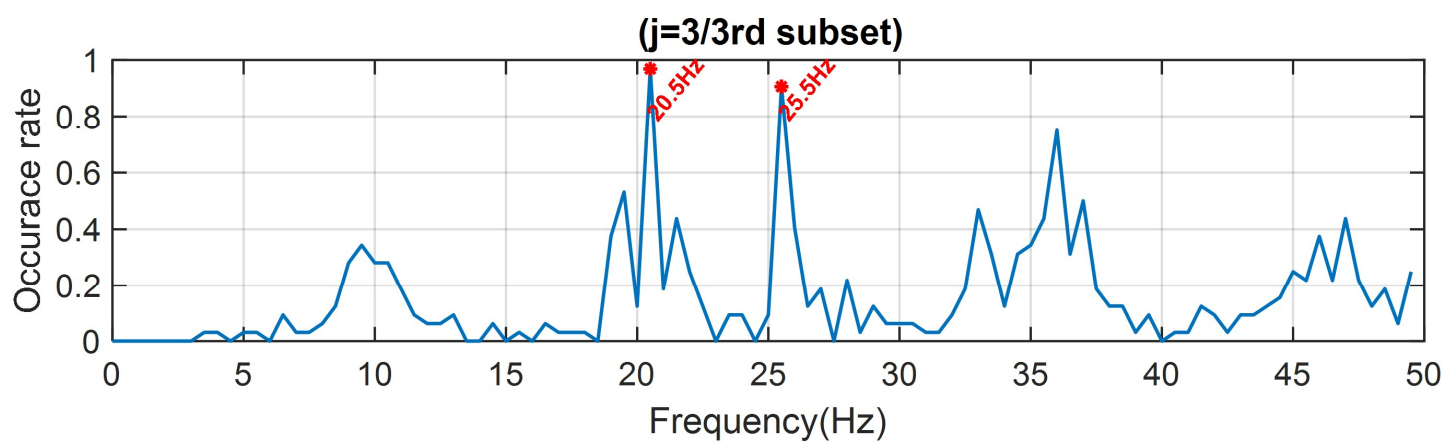

Figure 14 SD by CoS-SSI and the corresponding rate of stable rates 


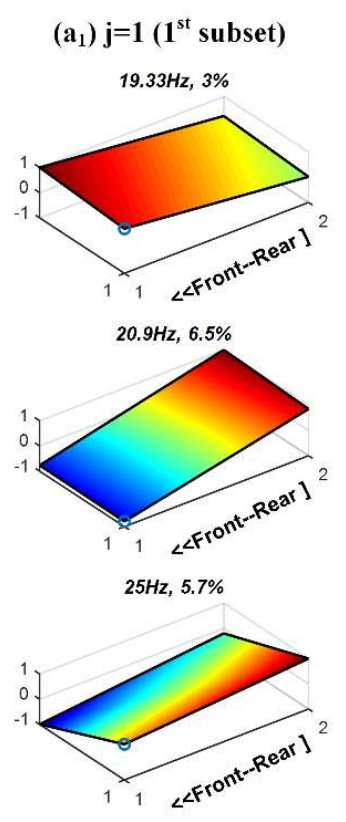

$\left(a_{2}\right) M \Lambda C$ of $j=1 / 1$ st subset
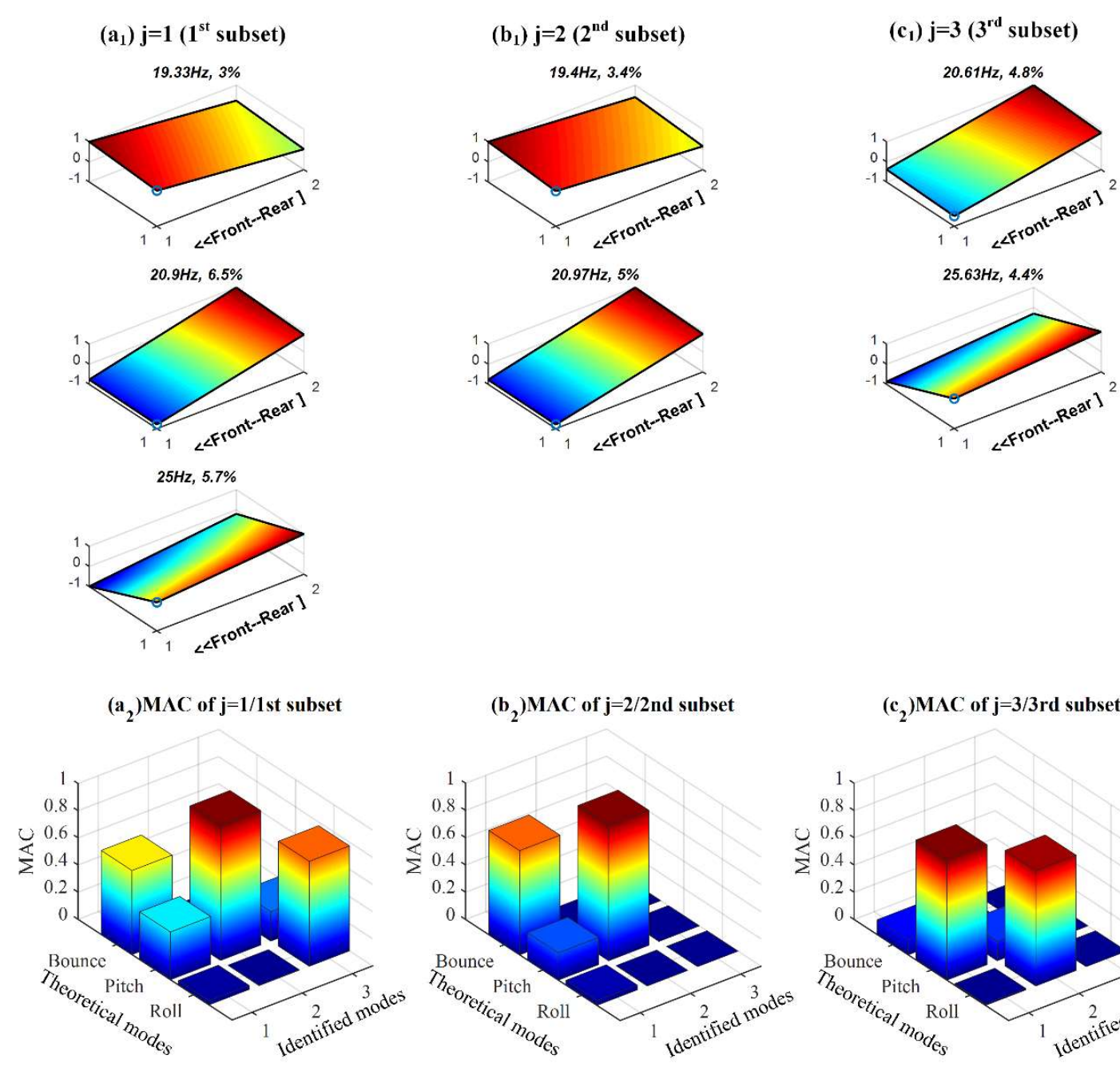

\begin{tabular}{r|}
${ }^{1}$ \\
0.8 \\
0.6 \\
0.4 \\
0.2 \\
0 \\
-0.2 \\
-0.4 \\
-0.6 \\
-0.8 \\
-1
\end{tabular}

$\left(c_{2}\right) M \Lambda C$ of $j=3 / 3$ rd subset

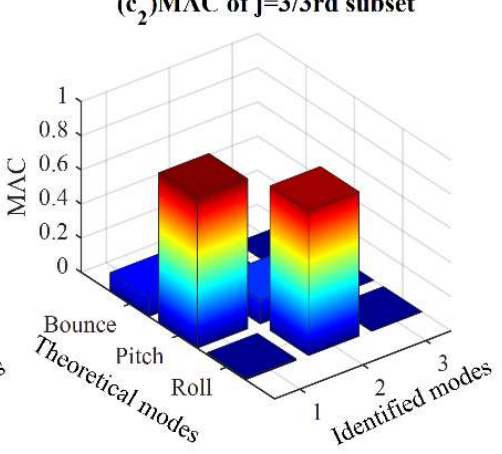

1
0.8
0.6
0.4
0.2
0

So far, all of the suspension related rigid modes of the bogie frame are identified by CoS-SSI. The identified modal parameters can be employed as the criterion to fulfil condition monitoring of the suspension systems since the rigid modes of the bogie frame are in rapport with the suspension system parameters. Moreover, the mode shapes could be employed as the main criterion because they are the inherent properties of dynamic systems. On the other hand, the frequencies could only be the secondary criterion since they may change with the system mass. The damping ratio can only be a reference because it cannot be identified accurately, which is a common issue in modal identification.

In addition, it took 29.56 seconds to get the final results using CoS-SSI method through a desktop computer with four Intel(R) Cores(TM) of i5-2310 CPU and 8G RAM. It is much shorter than the time taken to capture a full set of data which is as long as 400 seconds. The computational 
efficiency can be improved in a further step when the Matlab program is converted into a computationally efficient language such as $\mathrm{C}$ or $\mathrm{C}++$. Moreover, a lower sampling rate can also enhance the computational efficiency. These means that CoS-SSI method has the potential to fulfil the online monitoring.

\subsection{Modal properties of suspension system with faults}

In order to evaluate the performance and effectiveness of CoS-SSI method for suspension condition monitoring, two cases of suspension with faults were investigated in this section. The fault of suspension was artificially introduced by replacing the stud mounts at one corner. The employed stud mounts were tested on a universal test system to approximate their working stiffness beforehand.

\section{a) Stud mount test}

The employed mount bushings are shown in Figure 16. Three different kinds of male to male stud mounts with different rubber materials are used in this study. The three kinds of mount bushings have different diameters $(D)$, therefore, their stiffness and damping ratio will be different. However, their overall heights $(H)$ are the same to ensure the installation, which is $30 \mathrm{~mm}$. They were tested on a INSTRON 3369 universal testing system to obtain the force versus displacement curves, shown in Figure 16(e). In this test, all tested specimens were given the same maximum compressive extension $(3 \mathrm{~mm})$ under a load speed of $2 \mathrm{~mm} / \mathrm{min}$, and the corresponding forces were measured. Each specimen was loaded for three cycles and the force versus displacement curves are presented in Figure 17.

According to the force of mount bushing at $1 \mathrm{~mm}$, the stiffness of the harder stud mount is nearly double compared with the normal (reference) stud mount, and the stiffness of the softer stud mount is less than half of normal. According to the stiffness estimation method referred in Section 3.1, the stiffness of the faulty suspension can be estimated. Moreover, it can be seen from Figure 17 that the hysteresis characteristic of the employed stud mounts is apparent, which is one of the main reasons for the nonlinearity of the suspension system. 
(a) Schematic

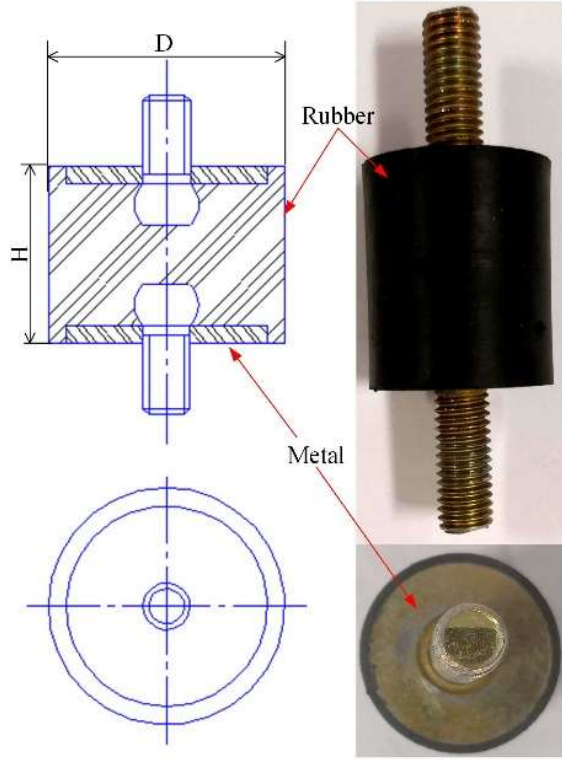

Figure 16 Employed stud mount and the stiffness test machine
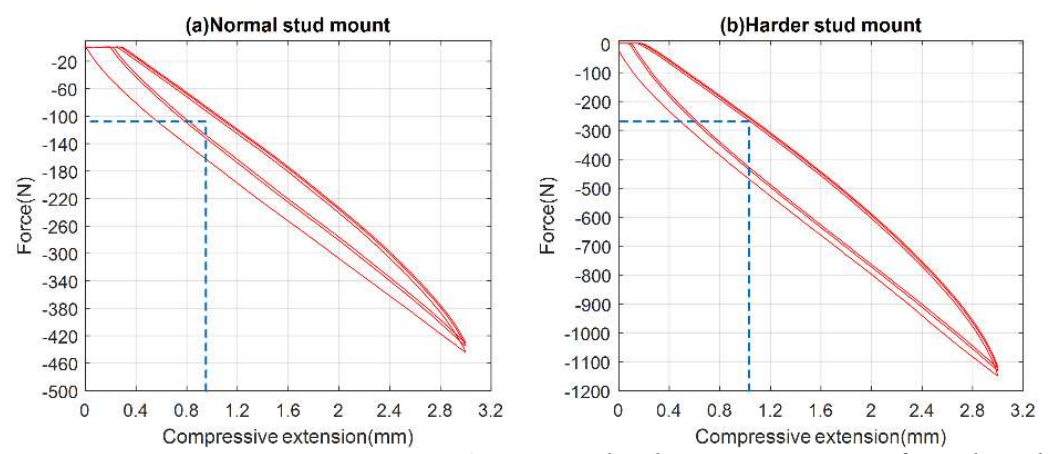

Figure 17 Force-displacement curves of employed stud mounts

\section{b) Fault case 1}

The first suspension fault case was replacing one stud mount at front-left (FL) with a harder one, which is to simulate the aging of the rubber. According to the force-displacement test of the stud mount, the stiffness of FL suspension will increase around 50\%. The other experimental conditions were the same as previously introduced in Section 3.2 and only the CoS-SSI was employed to identify the modal parameters.

The modal parameters identified from each subset are presented in Figure 18, in which MAC values are also compared with the theoretical results. It can be seen from Figure $18\left(\mathrm{a}_{1}\right),\left(\mathrm{b}_{1}\right)$, and $\left(\mathrm{c}_{1}\right)$ that all of the first modes identified from the three subsets have a smaller amplitude at the FL corner, which was caused from the stiffness change of FL suspension. Moreover, it can be seen 
from Figure 18( $\left.a_{2}\right),\left(b_{2}\right)$ and $\left(c_{2}\right)$ that the first mode identified from each subset should be bounce. Secondly, it is evident that the resonance frequency of pitch and roll modes increased about $2 \mathrm{~Hz}$ as the stiffness of FL suspension increased by $50 \%$. Based on these two characteristics, it can diagnose that the suspension system has faults at FL corner.
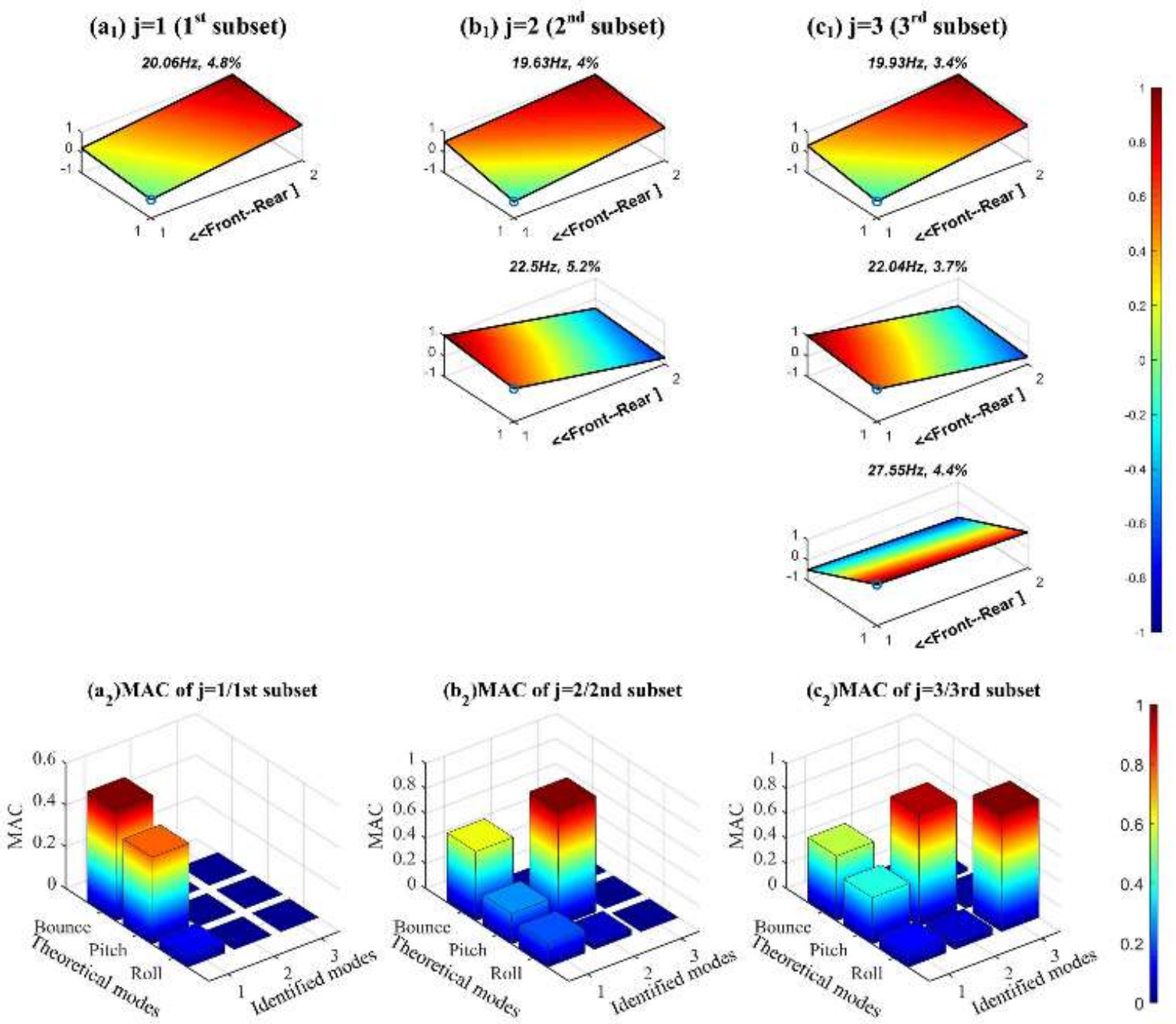

Figure 18 Identified modes along with MAC values for the softer suspension at FL corner

\section{c) Fault case 2}

The second fault case was replacing both two stud mounts at right left (RL) with two softer ones. As a consequence, the stiffness of RL suspension was reduced by $75 \%$ according to the forcedisplacement curves. The purpose of this case was to simulate the fatigue of the suspension components. The other experimental conditions were same as previously introduced in Section 3.2. The results identified by the CoS-SSI are presented in Figure 19. 
Firstly, it can be seen from Figure 19 that all of the identified modes have larger amplitudes at the $R L$ corner which were as a result of the stiffness reduction of the RL suspension. This characteristic compared well with the results identified from the previous fault case where the harder suspension resulted in smaller amplitude. Furthermore, it is noticeable that only pitch mode has been identified in the results. According to the MAC values in Figure $19\left(a_{2}\right),\left(b_{2}\right)$ and $\left(c_{2}\right)$, even the mode around $25 \mathrm{~Hz}$, which should be roll mode, looks like pitch mode. This could be another good indication of a fault in the suspension system.

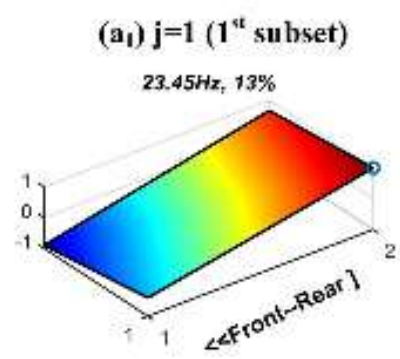

$24.54 \mathrm{~Hz}, 9.5 \%$

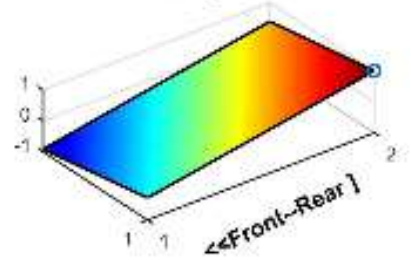

$\left(a_{2}\right)$ MAC of $\mathrm{j}=\mathrm{I} / 1$ st subset

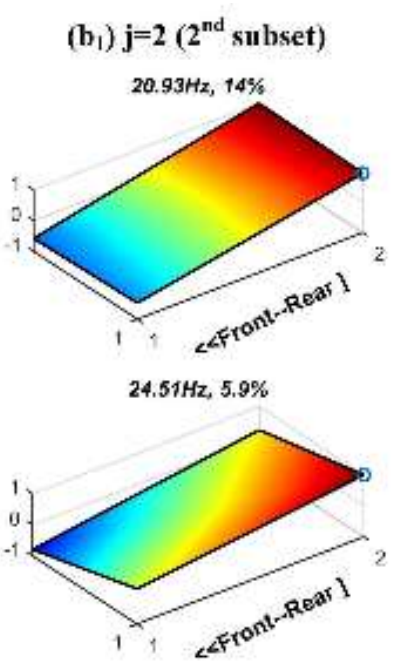

$\left(\mathrm{b}_{2}\right)$ MAC of $\mathrm{J}=2 / 2 \mathrm{nd}$ suloset

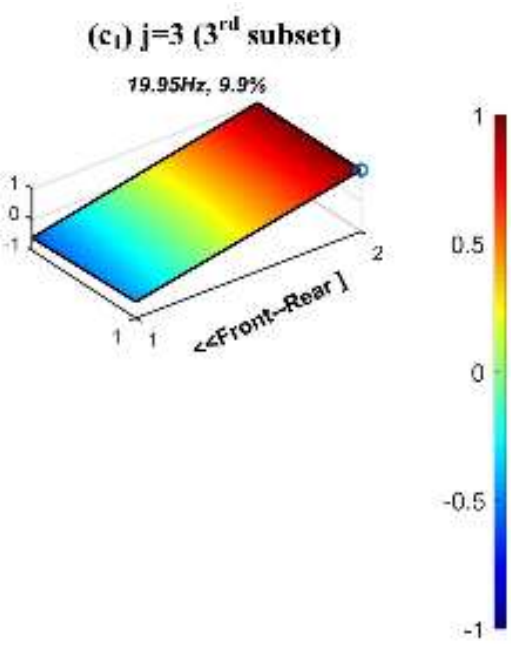

$\left(c_{2}\right)$ MLAC of $\mathbf{j}=\mathbf{3} / 3 \mathrm{rd}$ subset
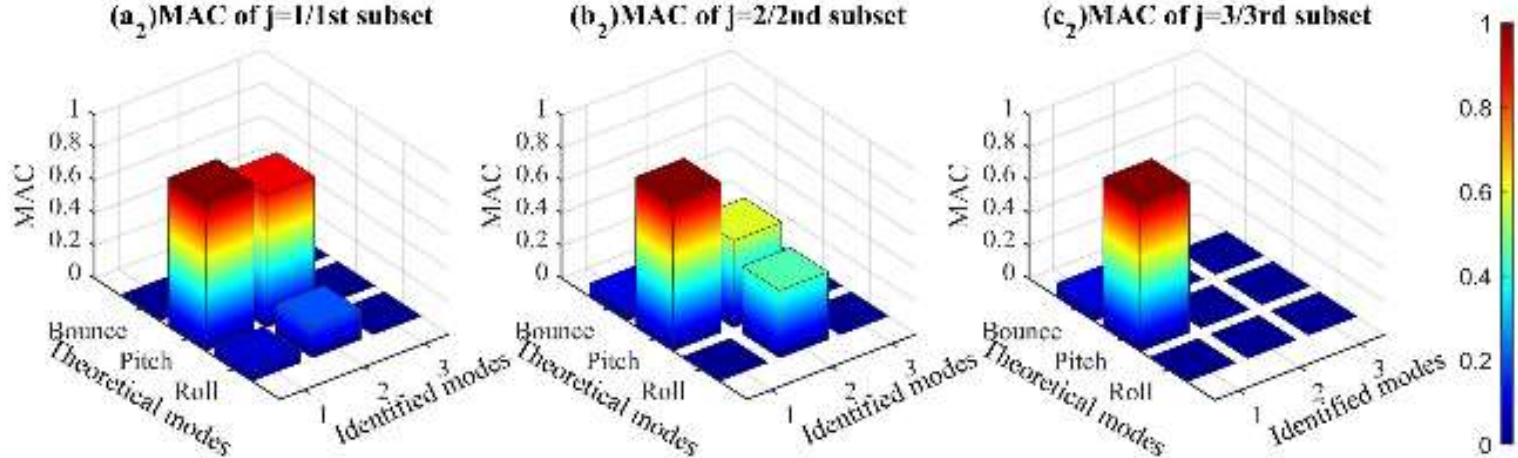

Figure 19 Identified modes along with MAC values for harder suspension at RL corner

\section{Conclusions}

The main aim of this paper is to try to develop a scheme to monitor the condition of railway vehicle suspension systems in real time. For this purpose, a novel OMA method, denoted as CoS-SSI, was proposed for vibration analysis by taking into account the inherent nonlinear and nonstationary of the suspension system. In order to evaluate the effectiveness of Cos-SSI, the experimental study 
was conducted on a $1 / 5^{\text {th }}$ scaled bogie operating on the roller rig to identify the modal parameters of bogie frame which are in relation to the suspension systems. Simultaneously, Cov-SSI and ACSSSI methods were employed to identify the modal parameters. Based on the identification results obtained from these three methods, the following conclusions can be drawn: CoS-SSI is much superior than Cov-SSI and ACS-SSI methods since it is the only method that has the ability to identify all rigid modes of bogie frame which are related to suspension systems. This indicated that CoS-SSI has the potential to achieve online monitoring of railway vehicle suspension systems by taking their nonlinearities into account. Moreover, two suspension faulty cases were investigated to evaluate CoS-SSI in a further step. The results indicated that CoS-SSI not only has the ability to identify the fault of suspension, but can also isolate the fault with high reliability.

\section{Acknowledgment}

The authors would like to thank the Hebei Provincial International Science and Technology Cooperation Program of China (Grant No.: 17394303D), the National Natural Science Foundation of China (Grant No.: 51605133; 51705127), China Scholarship Council (CSC Grant No.: 201608060041) for sponsorship and the Open Research Fund of the Traction Power State Key Laboratory, Southwest Jiaotong University, China (Grant No: TPL1704) to carry out the research.

\section{References}

1. Alemi, A., F. Corman, and G. Lodewijks, Condition monitoring approaches for the detection of railway wheel defects. Proceedings of the Institution of Mechanical Engineers, Part F: Journal of Rail and Rapid Transit, 2016: p. 0954409716656218.

2. Bruni, S., et al., Control and monitoring for railway vehicle dynamics. Vehicle System Dynamics, 2007. 45(7-8): p. 743-779.

3. Hussain, I., T. Mei, and R. Ritchings, Estimation of wheel-rail contact conditions and adhesion using the multiple model approach. Vehicle System Dynamics, 2013. 51(1): p. 32-53.

4. Li, C., et al., An overview: modern techniques for railway vehicle on-board health monitoring systems. Vehicle System Dynamics, 2017: p. 1-26.

5. Li, Y., et al., Fault detection method for railway wheel flat using an adaptive multiscale morphological filter. Mechanical Systems and Signal Processing, 2017. 84: p. 642-658.

6. Liang, B., et al., Railway wheel-flat and rail surface defect modelling and analysis by timefrequency techniques. Vehicle System Dynamics, 2013. 51(9): p. 1403-1421.

7. Mei, T. and X. Ding, Condition monitoring of rail vehicle suspensions based on changes in system dynamic interactions. Vehicle System Dynamics, 2009. 47(9): p. 1167-1181. 
8. Ngigi, R., et al. Modern techniques for condition monitoring of railway vehicle dynamics. in Journal of Physics: Conference Series. 2012. IOP Publishing.

9. Sun, Y.Q., C. Cole, and M. Spiryagin, Monitoring vertical wheel-rail contact forces based on freight wagon inverse modelling. Advances in Mechanical Engineering, 2015. 7(5): p. 1687814015585431.

10. Wei, X., H. Liu, and L. Jia. Fault detection of urban rail vehicle suspension system based on acceleration measurements. in Advanced Intelligent Mechatronics (AIM), 2012 IEEE/ASME International Conference on. 2012. IEEE.

11. Jesussek, M. and K. Ellermann, Fault detection and isolation for a full-scale railway vehicle suspension with multiple Kalman filters. Vehicle System Dynamics, 2014. 52(12): p. 1695-1715.

12. Liu, X., S. Alfi, and S. Bruni, An efficient recursive least square-based condition monitoring approach for a rail vehicle suspension system. Vehicle System Dynamics, 2016. 54(6): p. 814-830.

13. Peeters, B. and G. De Roeck, Reference-based stochastic subspace identification for output-only modal analysis. Mechanical systems and signal processing, 1999. 13(6): p. 855-878.

14. Reynders, E., et al., Uncertainty quantification in operational modal analysis with stochastic subspace identification: Validation and applications. Mechanical Systems and Signal Processing, 2016. 66: p. 13-30.

15. Priori, C., M. De Angelis, and R. Betti, On the selection of user-defined parameters in data-driven stochastic subspace identification. Mechanical Systems and Signal Processing, 2018. 100: p. 501-523.

16. Chen, Z., et al., Characterizing the dynamic response of a chassis frame in a heavy-duty dump vehicle based on an improved stochastic system identification. Shock and Vibration, 2015. 2015.

17. Chen, Z., et al., The average correlation signal based stochastic subspace identification for the online modal analysis of a dump truck frame. Journal of vibroengineering, 2015. 17(4): p. 1971-1988.

18. Guo, Y. and A. Kareem, System identification through nonstationary data using timefrequency blind source separation. Journal of Sound and Vibration, 2016. 371: p. 110-131.

19. Lin, C.-S., Ambient modal identification using non-stationary correlation technique. Archive of Applied Mechanics, 2016. 86(8): p. 1449-1464.

20. Lin, C.-S., D.-Y. Chiang, and T.-C. Tseng, An extended time series algorithm for modal identification from nonstationary ambient response data only. Mathematical Problems in Engineering, 2014. 2014.

21. James, G., T.G. Carne, and J.P. Lauffer, The natural excitation technique (NExT) for modal parameter extraction from operating structures. Modal Analysis-the International Journal of Analytical and Experimental Modal Analysis, 1995. 10(4): p. 260.

22. Xie, Y., P. Liu, and G.-P. Cai, Modal parameter identification of flexible spacecraft using the covariance-driven stochastic subspace identification (SSI-COV) method. Acta Mechanica Sinica, 2016. 32(4): p. 710-719.

23. Iwnicki, S.D. and A.H. Wickens, Validation of a MATLAB railway vehicle simulation using a scale roller rig. Vehicle System Dynamics, 1998. 30: p. 257-270.

(Total words: 6712) 